\title{
Quantum state discrimination on reconfigurable noise-robust quantum networks
}

\author{
Nicola Dalla Pozza $\odot^{*}$ and Filippo Caruso $\odot^{\dagger}$ \\ Department of Physics and Astronomy \& LENS, University of Florence, Via Carrara 1, I-50019 Sesto Fiorentino, Italy
}

(Received 25 March 2020; accepted 12 August 2020; published 2 October 2020)

\begin{abstract}
A fundamental problem in quantum information processing is the discrimination among a set of quantum states of a system. In this paper, we address this problem on an open quantum system described by a graph, whose evolution is defined by a quantum stochastic walk. In particular, the structure of the graph mimics those of neural networks, with the quantum states to discriminate encoded on input nodes and with the discrimination obtained on the output nodes. We optimize the parameters of the network to obtain the highest probability of correct discrimination. Numerical simulations show that after a transient time the probability of correct decision approaches the theoretical optimal quantum limit. These results are confirmed analytically for small graphs. Finally, we analyze the robustness and reconfigurability of the network for different set of quantum states and show that this architecture can pave the way to experimental realizations of our protocol as well as novel quantum generalizations of deep learning.
\end{abstract}

DOI: 10.1103/PhysRevResearch.2.043011

\section{INTRODUCTION}

In the last decade, quantum stochastic walks (QSWs) have been proposed as a model to generalize both quantum walks and classical random walks [1]. Their formulation arises from the need to extend quantum walks to open quantum systems, with the aim of incorporating decoherence effects that are inevitably present in a real physical system. In fact, the formerly proposed quantum version of random walks, i.e., the quantum walk (QW) [2-6], has been defined by a unitary evolution of the state, without taking into account incoherent effects. This allows the walker's position on a graph to be in a superposition of states, a property that has been exploited to show that QWs are universal for quantum computation [7-9] and that they allow to design quantum algorithms with computational advantages over classical algorithms [10-19].

In parallel to these results, there have been works showing the beneficial impact of decoherence for dephasing-enhanced transport in a variety of systems, in particular in lightharvesting complexes [20-26]. This has motivated the study of quantum walks with an environmental interaction, as is described by QSWs. The QSW framework has been investigated in the context of relaxing property [27-29] and propagation speed [30,31], showing advantages for speed-up in learning algorithms [32], and enhancement of excitation transport [33-37].

\footnotetext{
*nicola.dallapozza@unifi.it

†filippo.caruso@unifi.it
}

Published by the American Physical Society under the terms of the Creative Commons Attribution 4.0 International license. Further distribution of this work must maintain attribution to the author(s) and the published article's title, journal citation, and DOI.
The evolution of QSWs is defined by a GoriniKossakowski-Sudarshan-Lindblad master equation [38-40],

$$
\frac{d \rho}{d t}=-(1-p) i[H, \rho]+p \sum_{k}\left(L_{k} \rho L_{k}^{\dagger}-\frac{1}{2}\left\{L_{k}^{\dagger} L_{k}, \rho\right\}\right),
$$

where one assumes to work in units with $\hbar=1, H$ is the Hamiltonian, $\left\{L_{k}\right\}$ are the Lindblad operators, and both are defined from the adjacency matrix describing the network of nodes involved in the random walk. The smoothing parameter $p$ accounts for the amount of coherent evolution given from $H$ with respect to the irreversible evolution given by the Lindblad operators, and it allows one to interpolate between a quantum walk $(p=0)$ and a classical random walk $(p=1)$.

On the other side, quantum state discrimination has been one of the first problems faced in quantum information theory [41-48], but it is still a flourishing research field as demonstrated from recent theoretical [49-54] and experimental works [55-60], also considered in relation to machine learning approaches [61]. In its most general formulation, an observer wants to guess the quantum state of a system that is prepared in one of a set of feasible states, possibly by optimizing the measurement operators to apply on the system. The performance measure is the probability of correct detection

$$
P_{c}=\sum_{n=1}^{\mathcal{M}} p_{n} \operatorname{Tr}\left[\Pi_{n} \rho^{(n)}\right]
$$

where $\left\{\rho^{(n)}\right\}$ is the set of quantum states to discriminate, $n=1, \ldots, \mathcal{M},\left\{p_{n}\right\}$ their a priori probabilities, and $\left\{\Pi_{n}\right\}$ the measurement operators to estimate them.

In this work we consider the discrimination of quantum states as a result of their time evolution. Our structure is inspired by neural networks, with its evolution described by a quantum stochastic walk. The connection between the implementation of measurement operators (possibly to solve 
the discrimination problem) and quantum walks has been investigated in recent works $[62,63]$, but in these papers an alternative formulation of quantum walks is used, and we explicitly refer to a neural network structure for the quantum system. We test different sets of quantum states and several networks in order to understand the best topologies for the discrimination problem.

The paper is organized as follows. In Sec. II we review the formalism of quantum stochastic walks, and we introduce the network model that describes the quantum system. We present different topologies for the binary discrimination and for the discrimination between $\mathcal{M}>2$ quantum states. In Sec. III we formalize the discrimination problem, discussing in Sec. III A the binary case and in Sec. III B the $\mathcal{M}$-ary case. In Sec. IV we report the conclusions and final discussions.

\section{QUANTUM STOCHASTIC WALKS}

In this section we introduce the QSW model that we here apply to the discrimination problem.

Classical random walks, quantum walks, and quantum stochastic walks are usually defined on an graph $\mathcal{G}$, which is defined by a pair $\mathcal{G}=(\mathcal{N}, \mathcal{E})$, with $\mathcal{N}$ being a set of elements called nodes (or vertices) and $\mathcal{E}$ being a set of pairs of nodes $\left(\mathcal{N}_{i}, \mathcal{N}_{j}\right)$ representing arcs from $\mathcal{N}_{i}$ to $\mathcal{N}_{j}$. The pairs in $\mathcal{E}$ can be summarized in the adjacency matrix $A$, with

$$
A_{j, i}=\left\{\begin{array}{ll}
1, & \text { if }\left(\mathcal{N}_{i}, \mathcal{N}_{j}\right) \in \mathcal{E} \\
0, & \text { if }\left(\mathcal{N}_{i}, \mathcal{N}_{j}\right) \notin \mathcal{E}
\end{array} .\right.
$$

As a generalization, weighted graphs can have any real values $A_{j, i}$ assigned to an arc. Also, in the case the adjacency matrix is a symmetric matrix, i.e., $A_{i, j}=A_{j, i}$, the graph is called undirected, otherwise the graph is said to be directed. Undirected graphs have pairs $\left(\mathcal{N}_{i}, \mathcal{N}_{j}\right)$ and $\left(\mathcal{N}_{j}, \mathcal{N}_{i}\right)$ with the same weight on them, and in this case the arcs are also called edges or links.

The name random walk comes from the fact that a walker, starting from an initial node and moving around randomly according to the link connections, assumes a time-dependent probability distribution that can be predicted with this framework. In particular, in the case of an undirected graph with equal weights on the edges, we can define the transitionprobability matrix $T$ of the possible node transitions as $T=$ $A D^{-1}$, where $D$ is the diagonal degree matrix, with $D_{i, i}=$ $\sum_{j} A_{j, i}$ representing the number of nodes connected to $i$. The probability distribution of the node occupation, written as a column vector $\vec{q}(t)$, is evaluated for a discrete time $(t)$ random walk as

$$
\vec{q}(t+1)=T \vec{q}(t)
$$

and for a continuous time random walk as

$$
\frac{d \vec{q}}{d t}=(T-I) \vec{q} .
$$

In the quantum scenario, the nodes are associated with the elements of the site basis [34] (see Sec. II A for an extensive description of the network). The evolution of the system can be given by the Gorini-Kossakowski-Sudarshan-Lindblad master equation (1), with both the Hamiltonian $H$ and the Lindblad operators $\left\{L_{i, j}\right\}$ depending on the adjacency matrix defined on the graph. In some models of QSW [1,34], the Hamiltonian operator $H$ is defined from the adjacency matrix, i.e., $H=A$, and with the Lindblad operators depending on the transition matrix defined on the graph as $L_{i, j}=\sqrt{T_{i, j}}|i\rangle\langle j|$. With this approach, optimizing the coefficients of a weighted undirected adjacency matrix $A$ fixes the Hamiltonian $H$ and the Lindblad operators via $T=A D^{-1}$. Here we relax this assumption, and, once the adjacency matrix defines the topology of the network, we optimize $H$ and $T$ independently. More precisely, the coefficients $A_{i, j}$ are used to decide whether the corresponding $H_{i, j}$ and $T_{i, j}$ will be optimized (independently) or are set to zero. This approach physically corresponds to optimize independently the hopping rates in $H$ and the noise rates in $L$. Of course, to define proper transition probabilities the matrix $T$ must satisfy a set of constraints,

$$
0 \leqslant T_{i, j} \leqslant 1 \forall i, j, \quad \sum_{i} T_{i, j}=1 \forall j,
$$

while for a matter of simplicity and to reduce the amount of parameters involved we take $H$ to be any real symmetric matrix, $H_{i, j}=H_{j, i}$, with zero entries on the diagonal.

Moreover, in the graph some nodes may have a particular role. There is usually a starting node that identifies the initial position of the walker. There might be also sink nodes, i.e., nodes that can irreversibly trap the received population. The latter are connected to the rest of the network only through an arc connecting a sinker node in the network to the sink, preventing a transition in the reverse direction. In the QSW, this is obtained with a Lindblad operator $L_{n}=|n\rangle\left\langle s_{n}\right|$ for each sink, which is added on the right side of Eq. (1),

$$
\sum_{n=1}^{\mathcal{M}} \Gamma\left(2|n\rangle\left\langle s_{n}|\rho| s_{n}\right\rangle\langle n|-\left\{\left|s_{n}\right\rangle\left\langle s_{n}\right|, \rho\right\}\right),
$$

with $s_{n}$ being the index of the node connected to the $n$th sink $|n\rangle, 1 \leqslant n \leqslant \mathcal{M}$, that is, $\left|s_{n}\right\rangle$ is the sinker node of $|n\rangle$. Overall, the master equation for the density operator $\rho$ describing the system reads

$$
\begin{aligned}
\frac{d \rho}{d t}= & -(1-p) i[H, \rho]+p \sum_{i, j} L_{i, j} \rho L_{i, j}^{\dagger}-\frac{1}{2}\left\{L_{i, j}^{\dagger} L_{i, j}, \rho\right\} \\
& +\Gamma \sum_{n=1}^{\mathcal{M}} 2|n\rangle\left\langle s_{n}|\rho| s_{n}\right\rangle\langle n|-\left\{\left|s_{n}\right\rangle\left\langle s_{n}\right|, \rho\right\},
\end{aligned}
$$

and the population at the $n$th sink at time $t=\tau$ (corresponding to the total evolution time) can be evaluated as

$$
\rho_{n, n}(\tau)=\langle n|\rho(\tau)| n\rangle=2 \Gamma \int_{0}^{\tau} \rho_{s_{n}, s_{n}}(t) d t .
$$

From here on, we assume $\Gamma=1$ since this parameter is just a factor defining the timescale ( $\Gamma \tau$ is dimensionless).

\section{A. Network model}

To define the topology of the graph we mimic the structure of neural networks [64-66]. The latter are described by complex graphs where the nodes (resembling neurons) are grouped into input, hidden, or output layers. Input nodes are those where the data to be processed are set. Output nodes are those containing the results of the desired task. Hidden nodes 
represent intermediate steps in the elaboration. Note that in this work we use the term layers only to identify a cluster of nodes, such that the parallelism with the neural networks should be taken loosely.

In the quantum case a similar network could be physically realized with an ensemble of two-level systems, one for each node, and with a walker realized by a single quantum exciton moving around. Each node is then associated to the state $|i\rangle=$ $|0 \ldots 1 \ldots 0\rangle$, corresponding to have one excitation $(|1\rangle)$ at the $i$ th node and $|0\rangle$ elsewhere.

Then we classify the quantum-network nodes into input, intermediate, and output layers. For a $M-N-O$ network we mean that there are $M$ input nodes, $N$ intermediate, and $O$ output ones (see, for instance, Fig. 1). Multiple intermediate layers may also be present; for instance, a 2-6-5-4 network has two nodes in the input layer, six nodes in the first intermediate layer, five nodes in the second intermediate one, and four output nodes. The input nodes are associated to a subset of the Hilbert space, where we prepare the initial quantum (pure or mixed) state of the system, initially in the ground state (no excitons). The network will then evolve in time according to Eq. (8). Note that in general the number of input nodes $\mathrm{M}$ is not related to the number $\mathcal{M}$ of quantum states to discriminate. By default, each node in a layer is fully connected with all the nodes of the same layer and with all the nodes of the following one. Only in the output layer is each sink connected only from its sinker. We also consider different topologies by reducing the connections between nodes within the same layer. When we want to refer to a topology that is not the default one, we use " $r$ " to indicate that the connectivity is reduced, i.e., some links are removed. The output nodes are sink nodes where the population gets trapped. After the time evolution of the network dynamic, we measure the sink population to estimate the initial quantum state in the discrimination problem (see Sec. III).

\section{Models for binary discrimination}

In the case of binary discrimination, we first consider the $2 r-2 r-2$ model [see Fig. 1(a)]. This is probably the simplest model one can imagine, with two input nodes connected to two sinker nodes and two sinks, but with no links between nodes of the same layer. Then we consider some of its variants obtained adding some links, for instance, the $2-2 r-2$ model, where the input nodes are connected among themselves, the $2 r-2-2$ model, with an additional link between the sinkers, the 2-2-2 model, with both these links added, and a $2 r-4-2$ model, which has four intermediate nodes. By comparing the performances of these models we analyze the role of the added edges. In addition, we investigate the role of the intermediate layers optimizing the $2 r-2 r-\cdots-2$ model for an increasing number of intermediate fully connected layers. This latter model is represented in Fig. 1(b).

\section{Models for $\mathcal{M}$-ary discrimination}

In the case of $\mathcal{M}$-ary discrimination, we consider a setup with the same number of quantum states to discriminate as the input nodes, $\mathcal{M}=M$, and one with a larger number of quantum state, i.e., with $\mathcal{M}>M$. For instance, we

\section{INPUT INTERMEDIATE OUTPUT (SINK)}

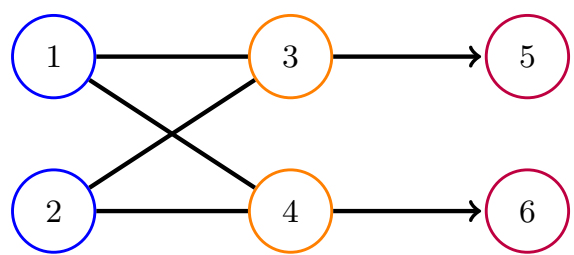

(a)

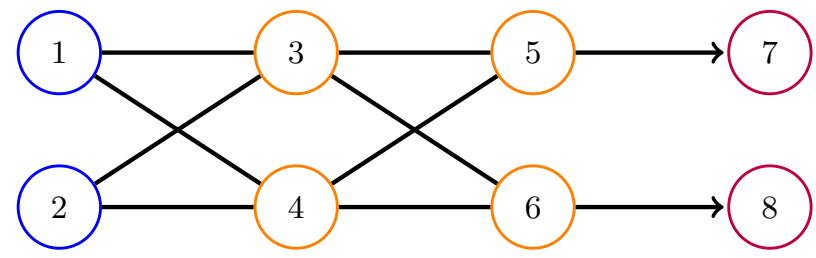

(b)

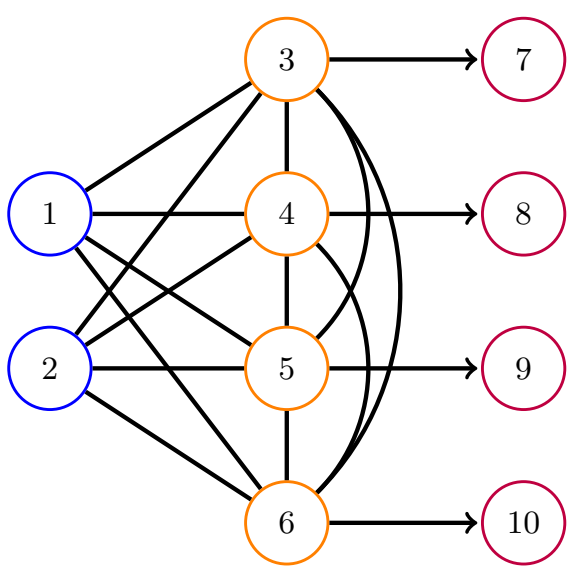

(c)

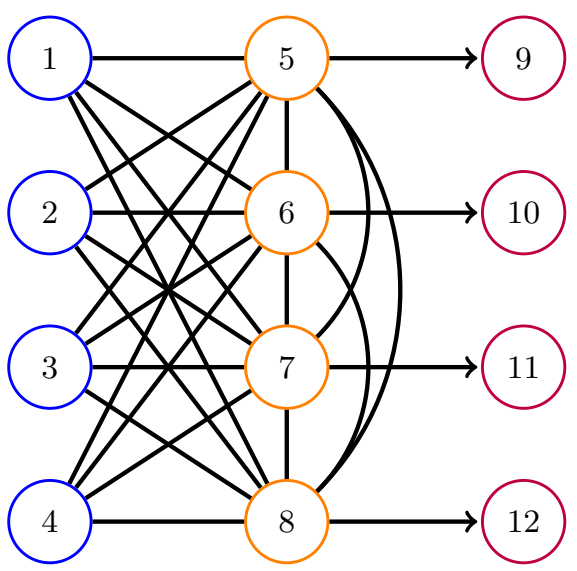

(d)

FIG. 1. Examples of $M-N-O$ network models: $2 r-2 r-2$ (a), $2 r-2 r-2 r-2$ (b), $2 r-4-4$ (c), and $4 r-4-4$ (d). In each panel, the left layer (blue) collects the input nodes and the right layer (purple) the output nodes, and between (orange) the intermediate nodes, which can be organized in multiple layers as in panel (b). Directed edges refer to irreversible transfer of population, while plain links indicate both coherent and incoherent transport. By default the nodes of a layer are fully connected within themselves. We indicate with $r$ when the connectivity is reduced, i.e., some links are removed. 
consider the $2-\mathcal{M}-\mathcal{M}, 2-\mathcal{M} r-\mathcal{M}$, and $2 r-\mathcal{M}-\mathcal{M}$ models [see Fig. 1(c)] for $\mathcal{M}=4$ and $\mathcal{M}=8$. As in the binary case, the reduction in the connectivity indicates the absence of links between nodes of the same layer. We also consider $\mathcal{M r}-\mathcal{M} r-\mathcal{M}, \mathcal{M r}-\mathcal{M}-\mathcal{M}$ [see Fig. 1(d)] and $\mathcal{M}-\mathcal{M}-\mathcal{M}$ models for $\mathcal{M}=4$.

\section{QUANTUM STATE DISCRIMINATION}

In this section we introduce the problem of quantum state discrimination (for more details see reviews [45-48]).

Assume that a quantum system is prepared in a quantum state drawn from a set of given states, represented by density operators $\left\{\rho^{(m)}, 1 \leqslant m \leqslant \mathcal{M}\right\}$ in the Hilbert space $\mathcal{H}$. The $a$ priori probabilities $p_{m}$ by which the quantum states are prepared are also known: $\left\{p_{m}, 1 \leqslant m \leqslant \mathcal{M}\right\}, \sum_{m} p_{m}=1$. In the discrimination problem, we search for the positive operatorvalued measurement (POVM) operators $\left\{\Pi_{n}, 1 \leqslant n \leqslant \mathcal{M}\right\}$ satisfying

$$
\Pi_{n} \geqslant 0, \quad \sum_{n=1}^{\mathcal{M}} \Pi_{n}=I_{\mathcal{H}},
$$

that allow us to estimate the prepared state with the highest probability of correct detection $P_{c}$, or equivalently, the lowest probability of error $P_{e}=1-P_{c}$. In Eq. (10) the term $I_{\mathcal{H}}$ represents the identity operator acting on the space of the density matrices of the quantum system $\mathcal{H}$.

If the quantum states $\left\{\rho^{(m)}\right\}$ span orthogonal subspaces, a perfect discrimination is possible by appropriate measurement operators and $P_{c}=1$. If this is not the case, the outcome corresponding to the measurement operator $\Pi_{n}$ may be correctly recorded when the prepared quantum state is $\rho^{(n)}$, or it may be wrongly recorded when the quantum state is $\rho^{(m)}, m \neq n$, leading to the probability of correct detection in Eq. (2).

The conditions for the optimal solution have been derived by Holevo [42] and by Yuen, Kennedy, and Lax [43]. In the most general scenario the problem can be solved numerically via semidefinite programming [67], but, in the binary case or if the set of quantum states exhibits symmetry features, the optimization can be further carried on analytically to better understand the structure of the measurement operators [68-70], and possibly to find a closed form for the probability of correct decision.

In the case of only two quantum states, the problem of binary discrimination has been solved by Helstrom [41], and the optimal probability of correct decision is known as the Helstrom bound. If the quantum states are pure, $\rho^{(1)}=$ $\left|\psi^{(1)}\right\rangle\left\langle\psi^{(1)}\right|$ and $\rho^{(2)}=\left|\psi^{(2)}\right\rangle\left\langle\psi^{(2)}\right|$, the Helstrom bound reads

$$
P_{c}^{\text {Helstrom }}=\frac{1}{2}\left(1+\sqrt{1-4 p_{1} p_{2}\left|\left\langle\psi^{(1)} \mid \psi^{(2)}\right\rangle\right|^{2}}\right) .
$$

If instead $\rho^{(1)}$ and $\rho^{(2)}$ are mixed, the Helstrom bound can be evaluated numerically [41]. When the discrimination is set among $\mathcal{M}>2$ quantum states, the theoretical optimal probability of correct decision $P_{c}^{*}$ is evaluated numerically, and it is used as a reference for the performance of the network.

Note that in our setup we actually consider an equivalent formulation of the problem, where instead of optimizing the measurement operators we fix the measurement projectors (on the population of the output nodes) and optimize the evolution of the network. The optimization of the evolution of the system concerns the coefficients of the Hamiltonian and the Lindblad operators to obtain the best evolution from the subspace of the input nodes, where the quantum states to discriminate are prepared, to the subspace of the output nodes, where the measurement is performed. For example, in the case of the discrimination of two qubits the time evolution "rotates" the quantum states such that the optimal projectors in the input subspace are now aligned with the projectors on the output subspace. In the discrimination of $\mathcal{M}>2$ qubits, the time evolution acts on a larger Hilbert space where to apply the $\mathcal{M}$ projectors corresponding to the sink node measurements (see Sec. III B). The two problems are equivalent, and one can interpret the optimized evolution with the measurement on the output nodes as realizing the Naimark extension of the POVM defined on the input nodes for the original discrimination problem. To be more precise, to have a resolution of the identity as in Eq. (10) we formally need to include a projection on the subspace outside the output nodes. This is necessary since part of the population can be trapped in the network [24,34]. The outcome associated to this extra projector is considered inconclusive for the discrimination.

We fix the measurement operators to be $\left\{\Pi_{n}=|n\rangle\langle n|\right\}$, with $n$ identifying the $n$th sink, $1 \leqslant n \leqslant \mathcal{M}$, which is associated with the estimation of the input quantum state $\rho^{(n)}$. The projector associated with the inconclusive output is $\Pi_{\text {inc }}=$ $I_{\mathcal{H}}-\sum_{n} \Pi_{n}$.

The probability of correct decision can then be written as

$$
\begin{aligned}
P_{c} & =\sum_{n=1}^{\mathcal{M}} p_{n} \operatorname{Tr}\left[\Pi_{n} \rho^{(n)}(\tau)\right]=\sum_{n=1}^{\mathcal{M}} p_{n} \rho_{n, n}^{(n)}(\tau) \\
& =2 \Gamma \sum_{n=1}^{\mathcal{M}} p_{n} \int_{0}^{\tau} \rho_{s_{n}, s_{n}}^{(n)}(t) d t,
\end{aligned}
$$

where we have defined $\rho_{i, j}^{(m)}(t)$ as

$$
\rho_{i, j}^{(m)}(t)=\left\langle i\left|\rho^{(m)}(t)\right| j\right\rangle .
$$

The optimization of the network coefficients has been performed numerically using standard routines employing an interior-point algorithm [71-74] to maximize the probability of correct decision $P_{c}$.

In the case of the simple network $2 r-2 r-2$ for the binary discrimination, we further carry on the optimization analytically and solve the problem for $p=0$ and $p=1$. This has given us some insights on how to interpret the behavior of the performance as a function of $p$ and $\tau$. More details are reported in Appendixes A-D.

\section{A. Binary discrimination}

We set up the discrimination problem with different pairs of states, for different values of $p \in[0,1]$ and of the total evolution time $\tau$ in Eqs. (8) and (9). For each pair $(p, \tau)$ we optimize $H, T$ in Eqs. (6) and (8) assuming equal a priori preparation probabilities of the states to get discriminated.

First, we consider a $2 r-2 r-2$ model. We discriminate between two pure states that are symmetric with 
respect to $|1\rangle$,

$$
\begin{aligned}
\left|\psi^{(1)}\right\rangle & =\cos \theta|1\rangle+\sin \theta|2\rangle, \\
\left|\psi^{(2)}\right\rangle & =\cos \theta|1\rangle-\sin \theta|2\rangle,
\end{aligned}
$$

and

$$
\begin{gathered}
\left|\psi^{\left(1^{\prime}\right)}\right\rangle=\cos \theta|1\rangle+i \sin \theta|2\rangle, \\
\left|\psi^{\left(2^{\prime}\right)}\right\rangle=\cos \theta|1\rangle-i \sin \theta|2\rangle,
\end{gathered}
$$

with $\theta=\pi / 8$. The probability of correct decision $P_{c}$ is shown as a function of $(p, \tau)$ in Figs. 2(a) and 2(b), with $\rho^{(1)}=$ $\left|\psi^{(1)}\right\rangle\left\langle\psi^{(1)}\left|, \quad \rho^{(2)}=\right| \psi^{(2)}\right\rangle\left\langle\psi^{(2)}\right|$ reported below each plot. We also consider the discrimination between the pure state $\left|\psi^{(1)}\right\rangle\left[\left|\psi^{\left(1^{\prime}\right)}\right\rangle\right]$ and a mixed state $\rho^{(2)}\left[\rho^{\left(2^{\prime}\right)}\right]$ with the same spherical coordinates $\left(r_{x}, r_{y}, r_{z}\right)$ in the Bloch sphere representation $\rho^{(2)}=\left(1+r_{x} \sigma_{x}+r_{y} \sigma_{y}+r_{z} \sigma_{z}\right) / 2$ but with a radius $r=\sqrt{r_{x}^{2}+r_{y}^{2}+r_{z}^{2}}$ reduced to 0.5 (this value has been chosen to be intermediate between that of a pure state and the completely mixed state). The plots are shown in Figs. 2(c) and 2(d). Then, we consider the discrimination between the mixed quantum states $\rho^{(1)}, \rho^{(2)}\left[\rho^{\left(1^{\prime}\right)}, \rho^{\left(2^{\prime}\right)}\right]$ obtained from $\left|\psi^{(1)}\right\rangle,\left|\psi^{(2)}\right\rangle\left[\left|\psi^{\left(1^{\prime}\right)}\right\rangle,\left|\psi^{\left(2^{\prime}\right)}\right\rangle\right]$ reducing both radii to 0.5 . The plots are shown in Figs. 2(e) and 2(f). Note that the quantum states $\left|\psi^{\left(1^{\prime}\right)}\right\rangle,\left|\psi^{\left(2^{\prime}\right)}\right\rangle, \rho^{\left(1^{\prime}\right)}, \rho^{\left(2^{\prime}\right)}$ are simply obtained by rotating $\left|\psi^{(1)}\right\rangle,\left|\psi^{(2)}\right\rangle, \rho^{(1)}, \rho^{(2)}$ in the Bloch sphere in order to change the $r_{x}$ coordinates into the $r_{y}$ coordinates. We find that for increasing values of $\tau$ the performance increases. This can be interpreted by the fact that an initial quantum state requires some time to reach the sinks. In addition, we can see an almost-constant negative slope in $p$ for a fixed $\tau$, with the quantum walk ( $p=0$ ) outperforming the general quantum stochastic walk with $p>0$. We can also notice that the performance seems to saturate asymptotically, for any $p$, approaching the Helstrom bound for $p=0$ in panels (a), (c), and (e). Panels (b), (d), and (f) also show a clear gap between the surface and the optimal theoretical bound.

We have further investigated this behavior solving the optimization problem analytically for $p=0$ and $p=1$. We provide the expression for the optimal $H, T$ in Appendixes A-C, and we prove that for $p=0$ asymptotically we can reach the Helstrom bound, while for $p=1$ we reach the theoretical classical bound, that is, the Helstrom bound evaluated on the quantum states with the coherences set to zero.

Now we give a sketch of the solution in the particular case $p=0$ and the discrimination between the pure quantum states (14), $\theta \in[0, \pi / 2]$, on a $2 r-2 r-2$ model. Assuming the Hamiltonian in the form

$$
H=\left(\begin{array}{cccc}
0 & 0 & h & h \\
0 & 0 & h & -h \\
h & h & 0 & 0 \\
h & -h & 0 & 0
\end{array}\right),
$$

we can evaluate the time evolution of the node population (more details are found in Appendix C) and the probability of correct decision results

$$
\begin{aligned}
P_{c}(\tau) & =\int_{0}^{\tau} \rho_{3,3}^{(1)}(t)+\rho_{4,4}^{(2)}(t) d t \\
& =\frac{1+\sin (2 \theta)}{2}\left\{1-e^{-\tau}\left[\frac{z \sinh (z \tau)+\cosh (z \tau)-1}{z^{2}}+1\right]\right\}
\end{aligned}
$$

with $z=\sqrt{1-8 h^{2}}$. The maximization of $P_{c}(\tau)$ for a finite $\tau$ requires the minimization of the term $f(z)=[z \sinh (z \tau)+$ $\cosh (z \tau)-1] / z^{2}$, which can be accomplished numerically. In the asymptotic limit of $\tau \rightarrow \infty$ the term in the brackets vanishes, and $P_{c}(\infty)$ equals the Helstrom bound evaluated on $\left|\phi_{1}\right\rangle,\left|\phi_{2}\right\rangle$.

While in the case of the quantum states (14) the optimized network approaches the optimal performance for $p=0$ in the asymptotic limit, this is not the case for any pair of quantum states. In fact, the $2 r-2 r-2$ model has an invariant subspace [24] not connected to any sink. An invariant subspace of a quantum system dynamics is a Hilbert subspace where the dynamics is confined, i.e., span of the eigenstates of $\mathcal{H}$ that are orthogonal to the output nodes. In the case of the $2 r-2 r-2$ model, the invariant subspace is present for any $p$ and includes the $y$ component of the state in the Bloch sphere, that is, the $r_{y}$ component of the quantum state starts in this invariant subspace, and its evolution remains trapped there. This means that the problem is equivalent to discern the quantum states after setting $r_{y}$ to zero, effectively projecting the quantum states in the $\left(\sigma_{x}, \sigma_{z}\right)$ plane of the Bloch sphere (see Fig. 2, right panels).

Second, we investigate the role of some links in the network performance, by evaluating the performance of some variants of the $2 r-2 r-2$ model. For instance, we add a link in the input layer ( $2-2 r-2$ model), a link in the intermediate layer ( $2 r-2-2$ model), a link in both layers (2-2-2 model), an intermediate layer $(2 r-2 r-2 r-2$ model), and some intermediate nodes in the same layer $(2 r-4-2$ model). We compare the performances in Fig. 3, where we optimize the discrimination between a pure state and a mixed state, both with some $r_{y}$ coordinates,

$$
\begin{aligned}
& \rho^{(1)}=\left|\psi^{(1)}\right\rangle\left\langle\psi^{(1)}|, \quad| \psi^{(1)}\right\rangle=\cos \theta|1\rangle+\sin \theta e^{-i \xi}|2\rangle, \\
& \rho^{(2)}=\frac{1-r \cos \xi \sin 2 \theta \sigma_{x}+r \sin \xi \sin 2 \theta \sigma_{y}+r \cos 2 \theta \sigma_{z}}{2},
\end{aligned}
$$

with $\theta=\pi / 8, \xi=\pi / 4, r=0.5$. These values have been chosen to have both $r_{x}$ and $r_{y}$ coordinates, with an intermediate radius between 1 (corresponding to a pure state) and 0 (corresponding to the completely mixed state). The probability of correct decision as a function of $(p, \tau)$ has the same general behavior of Fig. 2. For this reason, in Fig. 3 we plot $P_{c}$ for $p=0$ as a function of $\tau$ for the different models, along with the Helstrom bound. As in the previous comparison, the performance increase in $\tau$ and the saturation threshold can be clearly observed. Indeed, it is interesting to compare the saturation value among the models. The models $2 r-2 r-2$ and $2 r-2 r-2 r-2$ have similar performances, showing a gap with the Helstrom bound in the asymptotic value. This is due to the presence of invariant subspaces trapping the quantum 


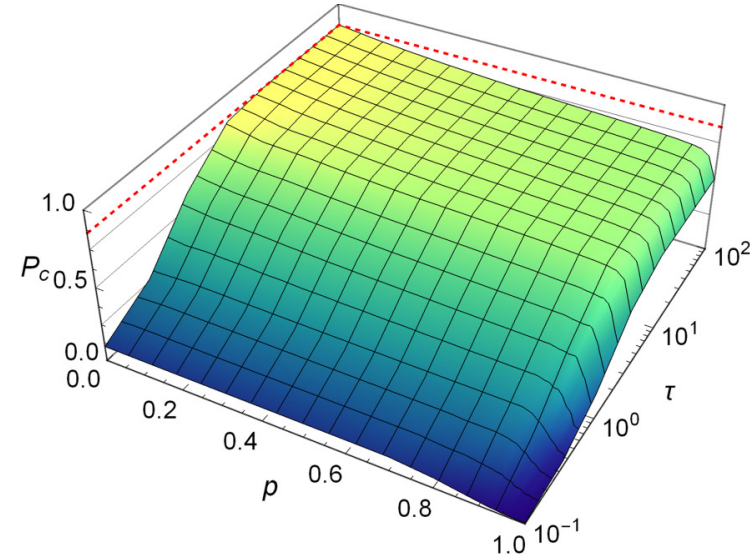

(a)

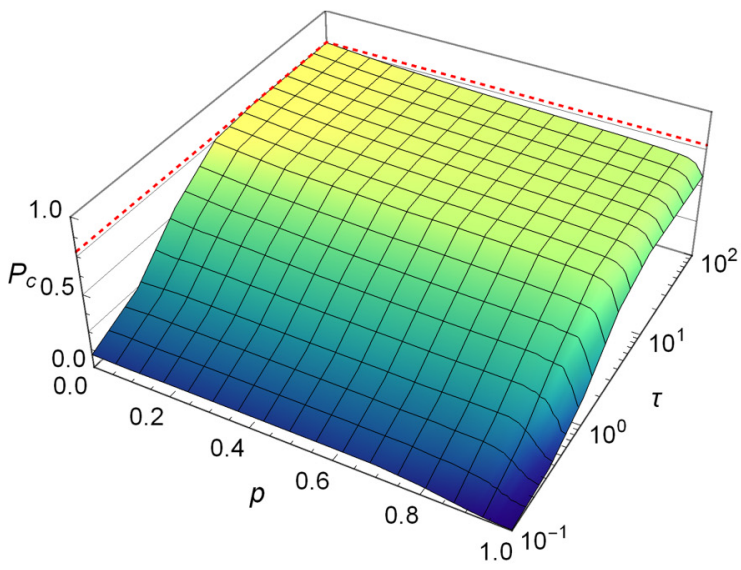

(c)

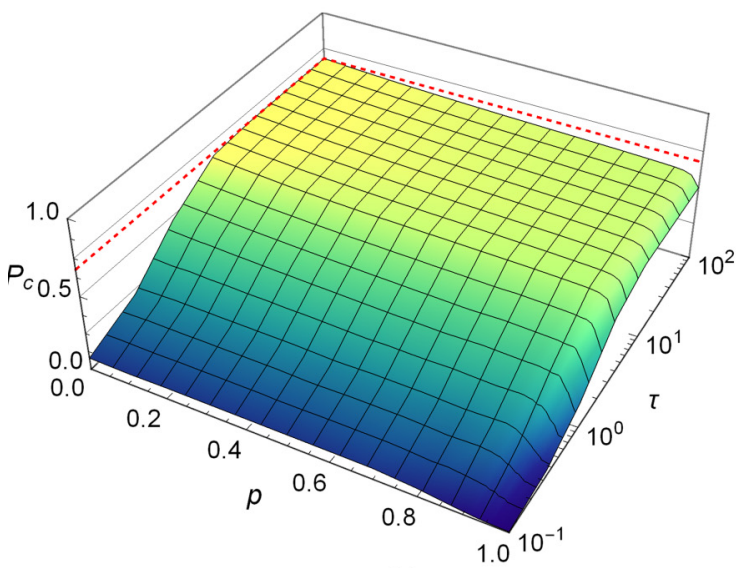

(e)

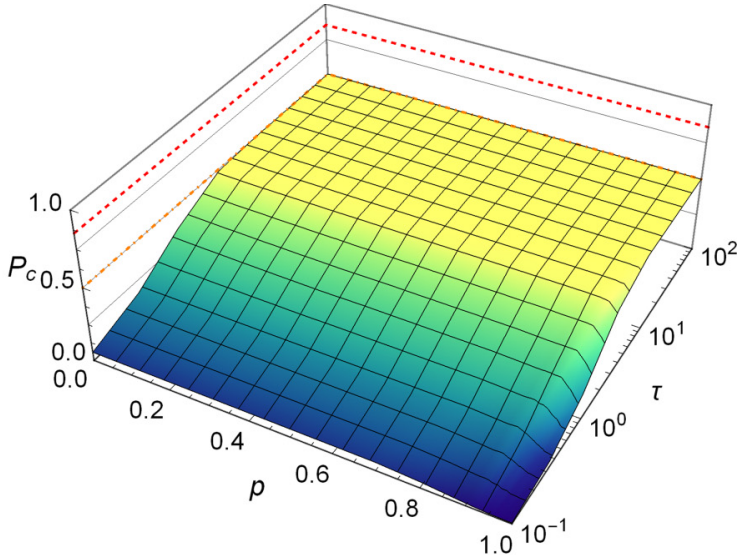

(b)

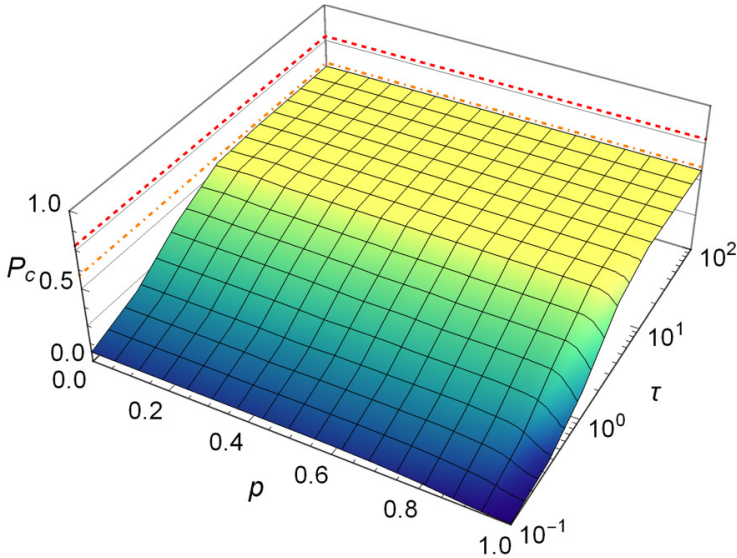

(d)

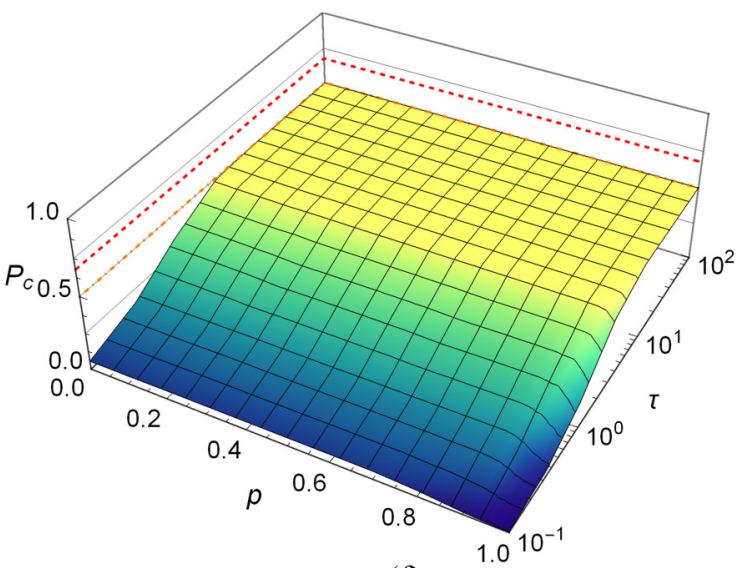

(f)

FIG. 2. Optimized probability of correct decision with respect to $(p, \tau)$ for the $2 r-2 r-2$ model in the case of binary discrimination with pure states (first row), a pure state and a mixed state (second row), and mixed states (third row). The quantum states, fully reported below, in the cases (a), (c), and (e) have $r_{x} \neq 0, r_{y}=0$, while the cases (b), (d), and (f) have been rotated to have the $r_{y}$ coordinates in place of the $r_{x}$ ones. The Helstrom bound is shown with a red dashed line, and with a dash-dotted orange line we evaluate the Helstrom bound on the same quantum states after setting $r_{y}=0$. The color map is linear between blue and yellow and normalized between the minimum and the maximum point in each plot. Following, we discriminate the quantum states:
(a) $\left(\begin{array}{l}0.8536 \\ 0.3536\end{array}\right.$
$\left.\begin{array}{l}0.3536 \\ 0.1464\end{array}\right)$ vs $\left(\begin{array}{c}0.8536 \\ -0.3536\end{array}\right.$
$\left.\begin{array}{c}-0.3536 \\ 0.1464\end{array}\right)$
(b) $\left(\begin{array}{c}0.8536 \\ i 0.3536\end{array}\right.$
$\left.\begin{array}{c}-i 0.3536 \\ 0.1464\end{array}\right)$ vs $\left(\begin{array}{c}0.8536 \\ -i 0.3536\end{array}\right.$
$\left.\begin{array}{c}i 0.3536 \\ 0.1464\end{array}\right)$
(c) $\left(\begin{array}{cc}0.8536 & -0.3536 \\ -0.3536 & 0.1464\end{array}\right)$ vs $\left(\begin{array}{l}0.6768 \\ 0.1768\end{array}\right.$
$\left.\begin{array}{l}0.1768 \\ 0.3232\end{array}\right)$
(d) $\left(\begin{array}{c}0.8536 \\ -i 0.3536\end{array}\right.$
$\left.\begin{array}{c}i 0.3536 \\ 0.1464\end{array}\right)$ vs $\left(\begin{array}{c}0.6768 \\ i 0.1768\end{array}\right.$
$\left.\begin{array}{c}-i 0.1768 \\ 0.3232\end{array}\right)$
(e) $\left(\begin{array}{c}0.6768 \\ -0.1768\end{array}\right.$
$\left.\begin{array}{c}-0.1768 \\ 0.3232\end{array}\right)$ vs $\left(\begin{array}{l}0.6768 \\ 0.1768\end{array}\right.$
$\left.\begin{array}{l}0.1768 \\ 0.3232\end{array}\right)$
(f) $\left(\begin{array}{c}0.6768 \\ i 0.1768\end{array}\right.$
$\left.\begin{array}{c}-i 0.1768 \\ 0.3232\end{array}\right)$ vs $\left(\begin{array}{c}0.6768 \\ -i 0.1768\end{array}\right.$
$\left.\begin{array}{c}i 0.1768 \\ 0.3232\end{array}\right)$ 


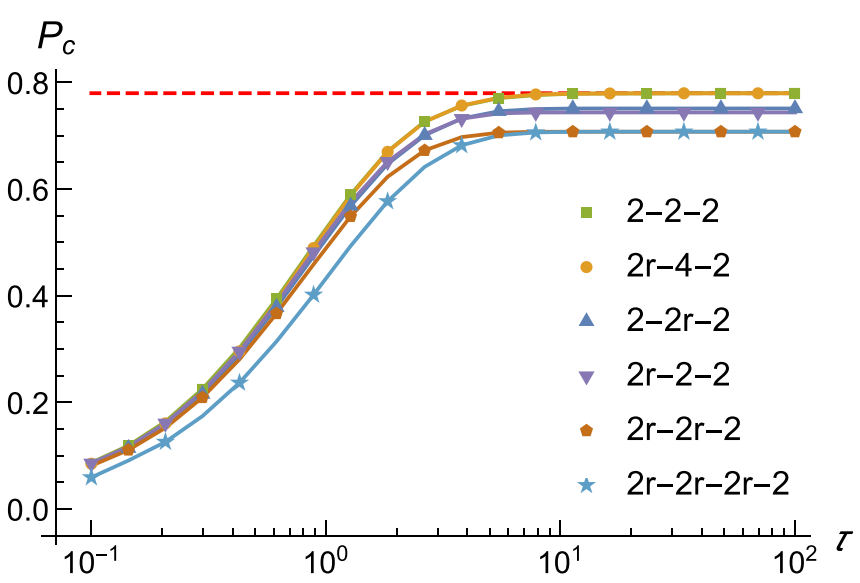

FIG. 3. Probability of correct detection $P_{c}$ for different variants of the $2 r-2 r-2$ model as a function of $\tau$ for $p=0$. The red dashed line shows the Helstrom bound for mixed quantum states in Eq. (18).

state component corresponding to the $r_{y}$ coordinates. Interestingly, the addition of a link in the input or intermediate layer of models $2 r-2-2$ and $2-2 r-2$, despite breaking the invariant subspaces, allow for an increase of the performance but do not close the gap with the Helstrom bound. Finally, the models $2-2-2$ and $2 r-4-2$ approach the upper bound. This is particularly interesting because it suggests that a reduced topology in the input nodes could be compensated by an increased number of intermediate nodes in a single layer.

We believe we cannot observe the beneficial impact of the noise since the graphs we consider are too small and simple where noise assisted transport is not present since interference effects are neutralized from static disorder in the Hamiltonian coefficients (see Refs. [24,33] and the references therein).

\section{Robustness}

Here we analyze the robustness of our discrimination scheme in the case of noisy preparation of the quantum states, a noisy configuration of the network, and an increasing number of intermediate layers.

In the former case, we optimize the 2-2-2 model assuming to discriminate the quantum states in Eq. (18) while only a noisy preparation is actually available, for instance, due to experimental imperfections in the preparation stage. We prepare the network with the optimal setup, i.e., with the optimal coefficients in $H, L$, but we input two random quantum states $\rho^{(1)}, \rho^{(2)}$ by uniformly sampling $\theta, \xi, r$ around their nominal value with a maximum $5 \%$ error. We run $10^{4}$ simulations with this setup as a function of $p$ for $\tau=1,10$, sampling new pairs of quantum states at each run. In Fig. 4 we show that even with a noisy preparation of the quantum states, for any $p$ the performances remain close to the theoretical values. Additionally, we focus on how the performance varies as a function of the preparation error ranging from $0 \%$ to $100 \%$ for $p=0$; see Fig. 5. We find that up to around a 5\% error the correct decision probability remains very close to the Helstrom bound and decreases linearly with the preparation error up to around $25 \%$, before exponentially dropping down below the random guess case (i.e., $P_{c}=0.5$ ).

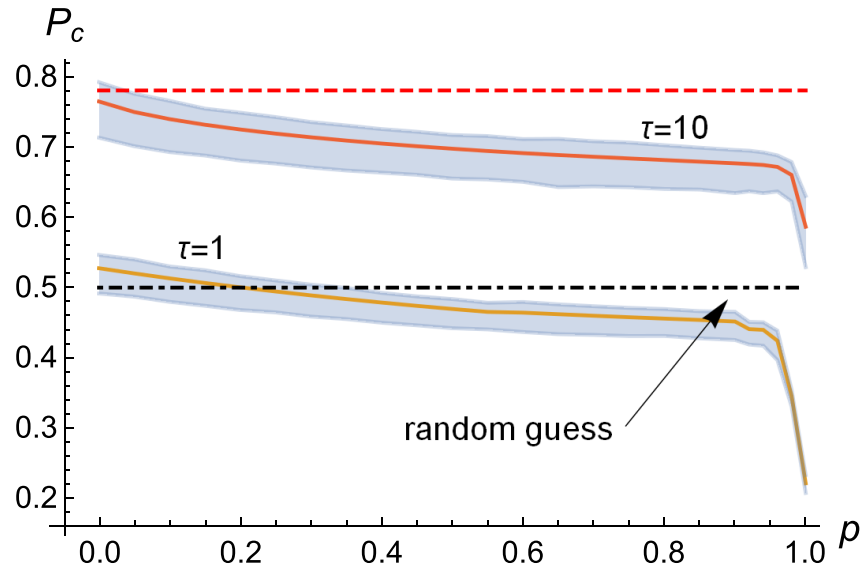

FIG. 4. Probability of correct decision $P_{c}$ (blue region) around the predicted performance (solid lines) with a noisy preparation of the quantum states to discriminate (5\% error). The red dashed line shows the Helstrom bound. For each $p$ and $\tau=1,10$ we run $10^{4}$ evaluations of $P_{c}$, each with a different pair of quantum states.

As a second robustness test, we consider a perfect preparation of the quantum states of Eq. (18) but a noisy setup of the network coefficients. In this case, we focus on $p=0,0.1$ and $\tau=1,10$, and in each simulation we sample the coefficients of the Hamiltonian $H$ uniformly around the optimal values with a given maximum percent error corresponding to a sort of network static disorder. Figure 6 shows that indeed the discriminatory network is robust against noisy preparations of the network dynamics, due, for instance, to experimental imperfections. It is also interesting to compare the two plots corresponding to the cases with $p=0$ and $p=0.1$. The former case achieves asymptotically a better performance with small network static disorder. The probability of correct decision slowly decreases as a function of this error and

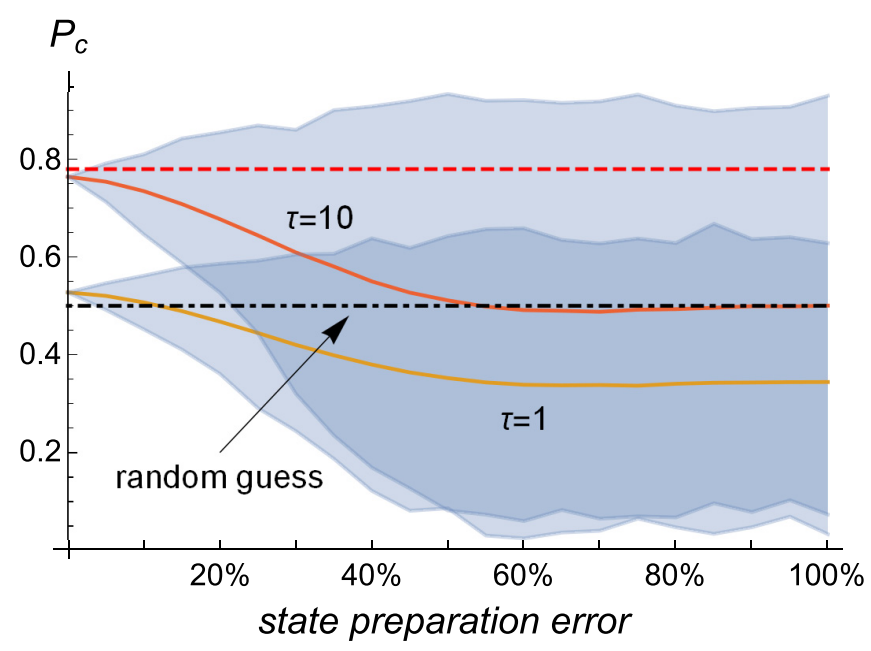

FIG. 5. Probability of correct decision $P_{c}$ (blue region) as a function of the maximum percent error on the preparation of quantum states, for $p=0$ and $\tau=1,10$. The continuous line shows the average performance over $10^{4}$ simulations. The black dash-dotted line shows the threshold corresponding to a random guess (0.5). The red dashed line shows the Helstrom bound. 


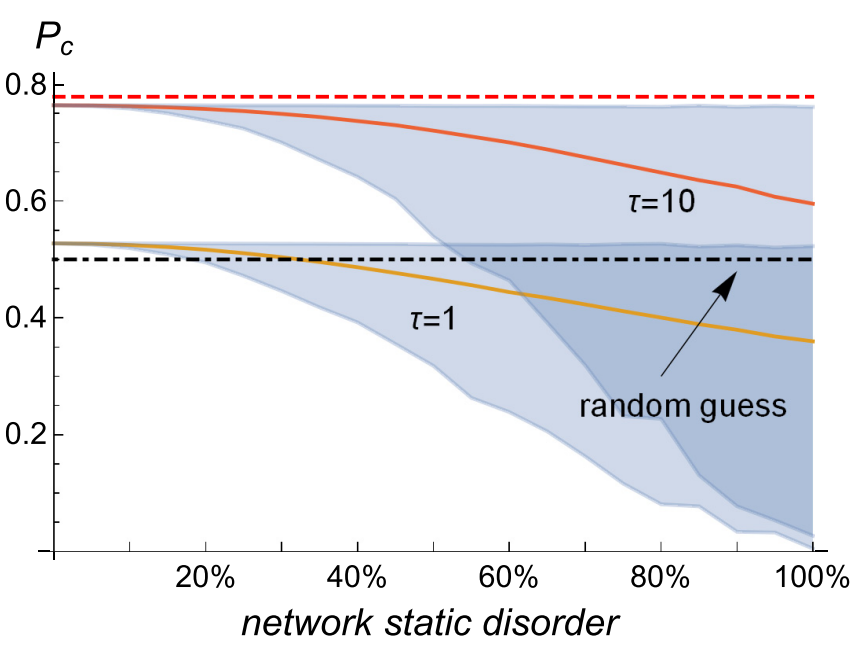

(a)

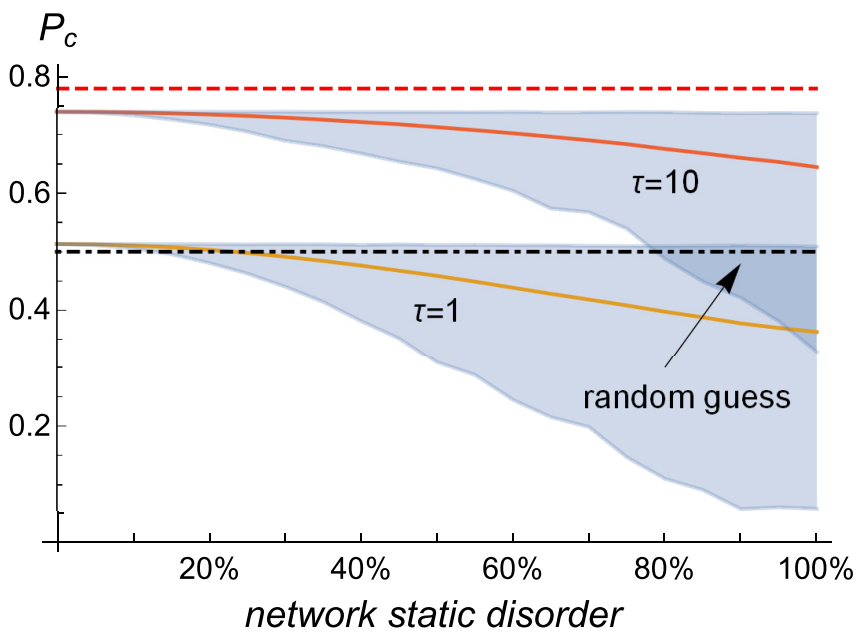

(b)

FIG. 6. Probability of correct decision $P_{c}$ (blue region) for a network prepared in a noisy configuration of its Hamiltonian coefficients $H_{i, j}$, as a function of its network static disorder percentage, for $p=0$ (a), $p=0.1$ (b) with $\tau=1, \tau=10$. The solid lines show the average performance over $10^{4}$ runs. The black dash-dotted line shows the threshold corresponding to a random guess (0.5). The red dashed line shows the Helstrom bound.

only at around a 50\% error it approaches the random guess limit. However, the $p=0.1$ case has a more robust performance with respect to this disorder, with the performance range crossing the threshold of random guess at around $80 \%$. Towards an experimental implementation of our protocol, it could be beneficial to consider $p=0.1$, slightly sacrificing the performances in favor of a more robust discrimination.

As a third robustness test we study whether the probability of correct decision improves adding or removing more intermediate layers. With $p=0$ we consider the models $2 r-2 r-2$ and $2 r-2-2$ with $1,2,4,8$, and 16 intermediate layers, and we plot the performance for different evolution times in Fig. 7. The two models, which have been tested with the quantum states of Fig. 2(d), present a lowering of the performance with the increase of the intermediate layers. This could be

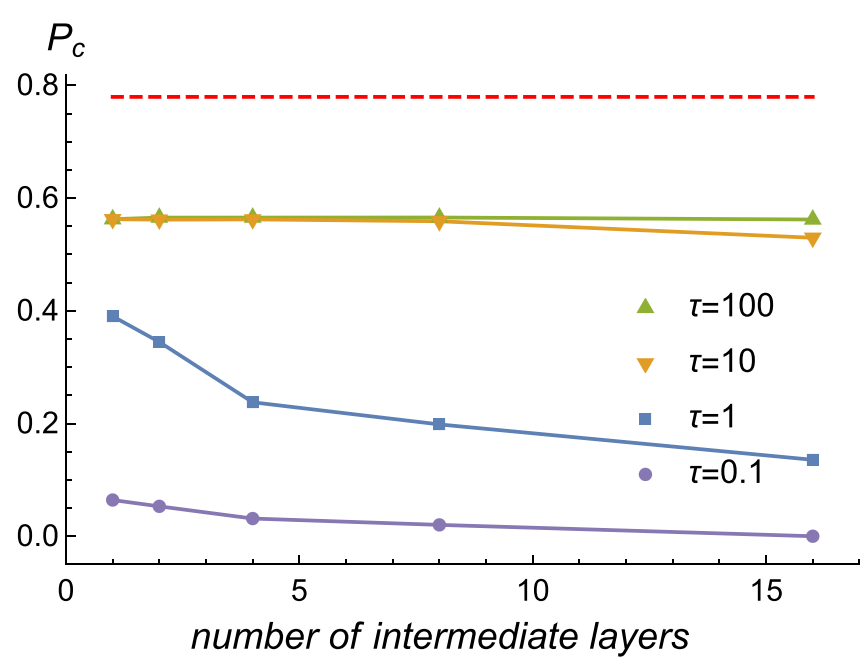

(a)

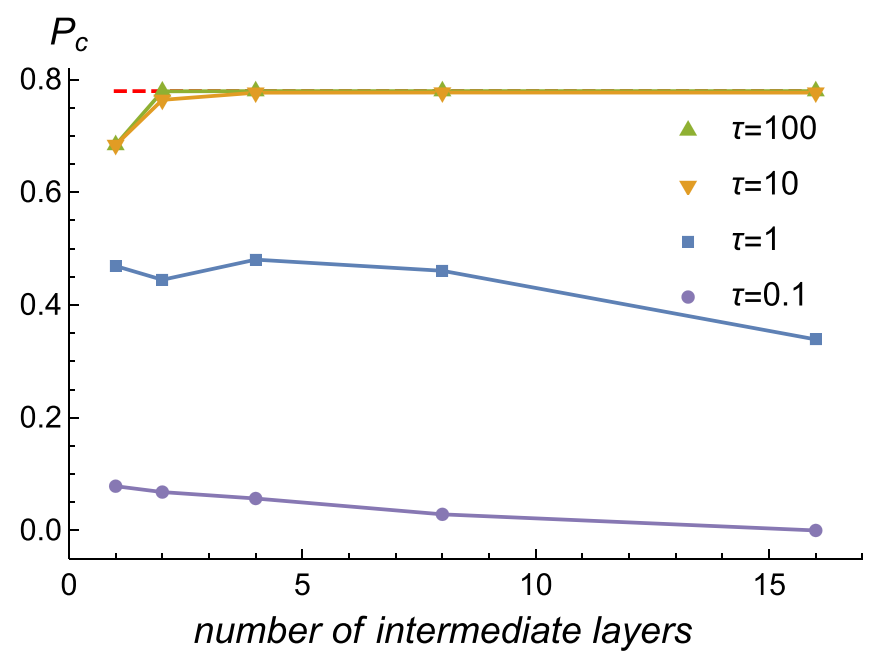

(b)

FIG. 7. Probability of correct decision between quantum states of Fig. 2(d) in the model $2 r-2 r-\cdots-2$ (a) and model $2 r-2-\cdots-2$ (b) as a function of the number of intermediate layers, for $\tau \in$ $\{0.1,1,10,100\}$. The (red) dashed line shows the Helstrom bound.

explained with the fact that as intermediate layers are added, the network requires more time to move the quantum states from the input nodes to the output nodes. However, while the increase of layers does not affect the asymptotic performance of model $2 r-2 r-2$, which keeps the gap with the Helstrom bound, in the model $2 r-2-2$ as soon as we add a second intermediate layer the performance approaches the optimal one. The increase in the connectivity is also beneficial at intermediate time $\tau$, with the performance of the latter model being greater already for $\tau=1$.

\section{B. $\mathcal{M}$-ary discrimination}

We now consider the generalization of our scheme to the discrimination of $\mathcal{M}$ quantum states. In particular, we investigate whether the number of nodes $M$ in the input layer poses limitations in the distinguishability of the quantum states. In 


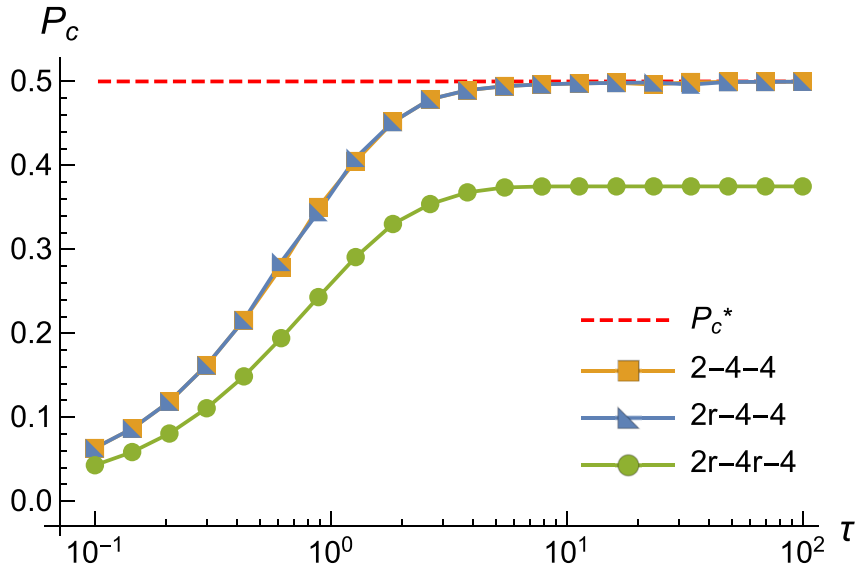

(a)

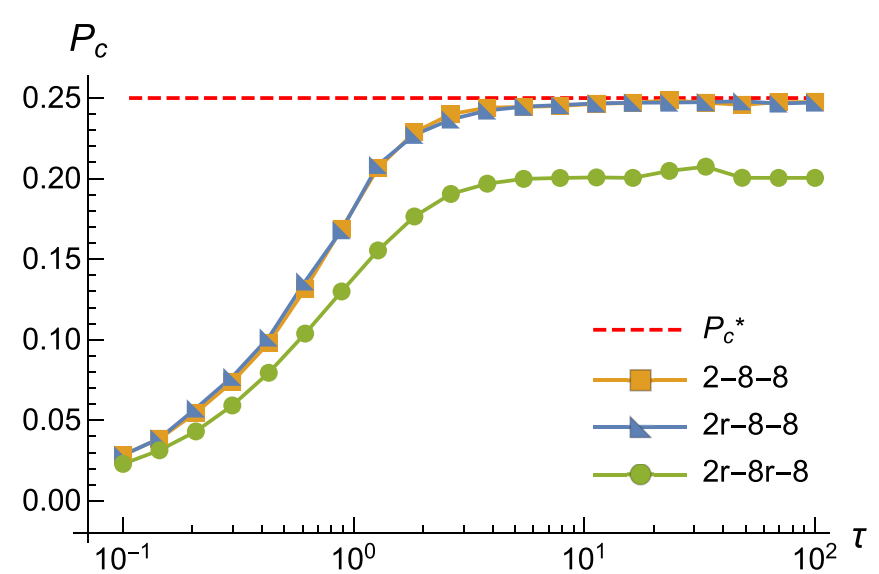

(b)

FIG. 8. Probability of correct detection $P_{c}$ of pure qubits (19) for $\mathcal{M}=4$ (a) and $\mathcal{M}=8$ (b) as a function of time $\tau$ for different variants of a $2-\mathcal{M}-\mathcal{M}$ model.

general, $M$ and $\mathcal{M}$ are not related, meaning that it could be $M<\mathcal{M}, M=\mathcal{M}$, or $M>\mathcal{M}$.

As a first case, we consider the discrimination of the $\mathcal{M}$ pure qubits $(M=2)$,

$$
\left|\psi^{(m)}\right\rangle=\frac{|1\rangle+e^{i 2 \pi \frac{m}{\mathcal{M}}}|2\rangle}{\sqrt{2}}, \quad m=1, \ldots, \mathcal{M},
$$

with $\mathcal{M}=4,8$. In the Bloch sphere representation, these quantum states are equally spaced along the equator defined by the $\operatorname{Tr}\left[\rho \sigma_{z}\right]=0$ plane, and because of this symmetry they are often used to test discrimination protocols [41,75].

We consider the models $2 r-\mathcal{M} r-\mathcal{M}, 2 r-\mathcal{M}-\mathcal{M}$ and $2-\mathcal{M}-\mathcal{M}$, whose performance are reported in Fig. 8, where we plot only the behavior for $p=0$ since the trend with respect to $p$ and $\tau$ is similar to the binary case. In this figure we just focus on understanding whether the topology asymptotically closes the gap with the optimal bound $P_{c}^{*}=1-2 / \mathcal{M}$, which has been reported, for instance, in Ref. [41].

There is, however, a fundamental difference here with respect to the binary case. While in the latter the optimal measurement operators are projectors, here the optimal ones are given by POVM. We can realize these POVM via projectors in

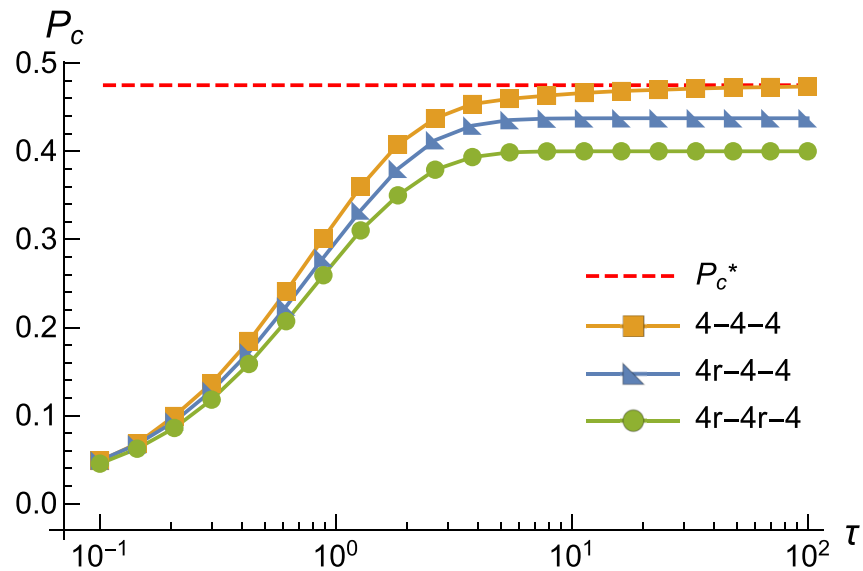

(a)

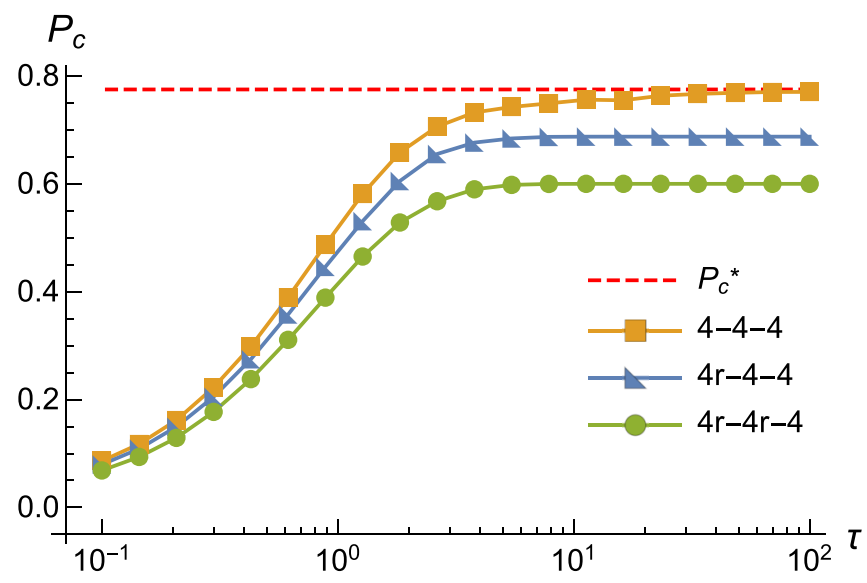

(b)

FIG. 9. Probability of correct detection $P_{c}$ for $\mathcal{M}=4$ as a function of time $\tau$ for different variants from a 4-4-4 model. The quantum states to discriminate are a mixture of a pure state and the completely mixed state with a factor $\alpha, 1-\alpha$ as in Eq. (20), with $\alpha=0.3$ (a) and $\alpha=0.7$ (b).

an extended Hilbert space using the Naimark theorem [41,7577], meaning that the optimal network will try to implement such extended projectors via its dynamics and the measurement on the sink nodes. We find that $2-\mathcal{M}-\mathcal{M}$ is the most general model and has the highest performance, approaching asymptotically the optimal $P_{c}^{*}$. Interestingly, the model $2 r-\mathcal{M}-\mathcal{M}$ share the same behavior, while for $2 r-\mathcal{M} r-\mathcal{M}$ the performances are clearly lower.

As a second case, we consider the discrimination with $\mathcal{M}=M$. We define the initial quantum states as a linear combination of pure states and the completely mixed state,

$$
\rho^{(m)}=\alpha\left|\varphi_{m}\right\rangle\left\langle\varphi_{m}\right|+(1-\alpha) \frac{I}{\mathcal{M}}, \quad m=1, \ldots, \mathcal{M},
$$

with $\left|\varphi_{m}\right\rangle$ being the $m$ th state in the mutually unbiased basis of the input nodes,

$$
\left|\varphi_{m}\right\rangle=\frac{1}{\sqrt{\mathcal{M}}} \sum_{k=1}^{\mathcal{M}} e^{-i \frac{2 \pi m k}{\mathcal{M}}}|k\rangle,
$$


where $|k\rangle$ is the quantum state associated with the $k$ th input node. For $\alpha=1$ it leads to discrimination of the pure orthogonal states $|k\rangle$. On the other hand, $\alpha=0$ means that $\rho^{(m)}=I / \mathcal{M}$ for all $m$, resulting in a completely random estimation with $P_{c}^{*}=1 / \mathcal{M}$. With an intermediate value of $\alpha$ we want to simulate a noisy preparation of the states (21). We consider $\alpha=0.3$ and $\alpha=0.7$, and we show the performance for different variants of the model $4-4-4$ in Fig. 9. The behavior of the probability of correct detection as a function of $p$ and $\tau$ is similar to the binary case. In the case of the model 4-4-4 we approach asymptotically the optimal theoretical bound for $p=0$, while for the models $4 r-4-4$ and $4 r-4 r-4$ there is a gap which is more or less emphasized depending on the value of $\alpha$.

\section{CONCLUSIONS}

In this work we have applied the formalism of quantum stochastic walks on configurable networks to the problem of quantum state discrimination, inspired by the neural network approach for deep learning of classical information as images In particular, the input nodes encode the quantum states to discriminate, while the output nodes are used to guess the right answer.

We test the discrimination of binary and $\mathcal{M}$-ary set of quantum states with multiple topologies, optimizing the coefficients of the Hamiltonian and the Lindblad operators to obtain the maximum probability of correct detection. The reconfigurability of the network architecture allows us to optimally discriminate numerous sets of quantum states. We observe that the general trend of the performances is to increase with the total evolution time $\tau$, while for a fixed $\tau$ the best performances are obtained lowering $p$. Notice that we are not observing any beneficial noise effects probably because the considered networks are very small and not homogeneous (not equal Hamiltonian and noise terms). In many cases with a pure quantum walk $(p=0)$ we can asymptotically approach the optimal theoretical performance. When this happens, the optimized dynamics realize the Naimark extension (on the whole quantum system) of the optimal POVM for the discrimination. In some cases there is a gap between the theoretical and the asymptotic performance for two reasons, i.e., the lack of node connectivity, which prevents the realization of the optimal POVM, or the presence of an invariant subspace trapping a portion of the quantum states to discriminate, which prevents this component from reaching the output nodes.

The role of the connectivity between nodes is highlighted in the Fig 3, where we have tested variations of a simple network by adding and removing links in the input and intermediate layer, and in Fig 7, where we have added more intermediate layers. Overall, the increase in the connectivity of the intermediate layer (or layers) leads to greater performances, possibly closing the gap with the Helstrom bound.

We have also analyzed the robustness of the optimized network with respect to the preparation of quantum states and the setup of the optimal coefficients of the Hamiltonian and Lindblad operators. Indeed, the architecture is very robust with respect to noise on both stages, as shown in Figs. 4 and 5. This analysis is promising for an experimental realization of the protocol, where imperfections in the preparation apparatus or in the network would be mitigated by the robustness of the architecture.

Therefore, we believe our results may represent further steps towards quantum implementations of machine learning protocols, for instance, to solve classification problem. Further studies will address the model applicability beyond discrimination problems, with larger networks, and using experimental benchmarks on photonics-based architectures and in cold atom platforms.

\section{ACKNOWLEDGMENTS}

This work was financially supported by Fondazione CR Firenze (projects Q-BIOSCAN and Quantum-AI), by PATHOS EU H2020 FET-OPEN Grant No. 828946, and by the University of Florence Grant No. Q-CODYCES.

\section{APPENDIX A: VECTORIZATION OF THE MASTER EQUATION}

In the following Appendixes we analytically evaluate and optimize the time evolution of the quantum system. This allows us to get a better insight on the performance of the optimization and to explain the asymptotic behavior in the small topologies.

We start by recalling the master equation that describes the evolution of a stochastic quantum walk on a graph connected to some sinks,

$$
\begin{aligned}
\dot{\rho}=- & (1-p) i[H, \rho]+p \sum_{i, j} L_{i, j} \rho L_{i, j}^{\dagger}-\frac{1}{2}\left\{L_{i, j}^{\dagger} L_{i, j}, \rho\right\} \\
& +\Gamma \sum_{n=1}^{\mathcal{M}} 2|n\rangle\left\langle s_{n}|\rho| s_{n}\right\rangle\langle n|-\left\{\left|s_{n}\right\rangle\left\langle s_{n}\right|, \rho\right\} .
\end{aligned}
$$

Equation (A1) defines a system of linear ordinary differential equations on the entries of the density matrix $\rho_{m, n}^{(x)}=$ $\left\langle m\left|\rho^{(x)}\right| n\right\rangle$. This conversion can easily be seen by applying the vectorization operation (by columns) on the members of (A1), exploiting the linear algebra property [78]

$$
\operatorname{vec}[A B C]=\left(C^{T} \otimes A\right) \operatorname{vec}[B],
$$

where $A, B, C$ are matrices with appropriate size. With the substitution $L_{i, j}=\sqrt{T_{i, j}}|i\rangle\langle j|$ we obtain

$$
\begin{aligned}
\operatorname{vec}[\dot{\rho}]= & {\left[-(1-p) i\left(I \otimes H-H^{T} \otimes I\right)\right.} \\
& +p \sum_{i, j} T_{i, j}|i\rangle\langle j|\otimes| i\rangle\langle j| \\
& -p \sum_{i, j} T_{i, j} \frac{1}{2}(I \otimes|j\rangle\langle j|+| j\rangle\langle j| \otimes I) \\
& +\Gamma \sum_{n} 2|n\rangle\left\langle s_{n}|\otimes| n\right\rangle\left\langle s_{n}\right| \\
& -I \otimes|n\rangle\langle n|-| n\rangle\langle n| \otimes I] \operatorname{vec}[\rho] \\
= & \tilde{L} \operatorname{vec}[\rho],
\end{aligned}
$$


where $\tilde{L}$ is the matrix that collects all the terms in the square brackets.

The items of the density matrix are collected in a (column) vector, i.e., $\operatorname{vec}[\rho]=\left[\rho_{1,1} \rho_{1,2} \ldots \rho_{m, n} \ldots\right]^{T}$, one can apply an invertible transformation $v=P \operatorname{vec}[\rho]$ that separates the real and imaginary part of the off-diagonal entries, $\rho_{m, n}=a_{m, n}+$ $i b_{m, n}$ with $m \neq n$, also rearranging the order of the items by putting the diagonal term first. This decomposition allows us to rewrite Eq. (A8) in terms of $r$ as

$$
\dot{v}=\left(P \tilde{L} P^{-1}\right)(P \operatorname{vec}[\rho])=\mathcal{L} v .
$$

\section{APPENDIX B: INVARIANT SUBSPACES}

In what follows we assume a $2 r-2 r-2$ model, and we keep track of only the entries of $\rho$ corresponding to input and intermediate nodes since the population on the sinks can be evaluated from Eq. (9). We define the Hamiltonian as

$$
H=\left(\begin{array}{cccc}
0 & 0 & h_{1} & h_{2} \\
0 & 0 & h_{3} & h_{4} \\
h_{1} & h_{3} & 0 & 0 \\
h_{2} & h_{4} & 0 & 0
\end{array}\right), \quad h_{k} \in \mathbb{R}
$$

and the transition matrix as

$$
T=\left(\begin{array}{cccc}
0 & 0 & t_{1} & t_{2} \\
0 & 0 & 1-t_{1} & 1-t_{2} \\
t_{3} & t_{4} & 0 & 0 \\
1-t_{3} & 1-t_{4} & 0 & 0
\end{array}\right), \quad 0 \leqslant t_{k} \leqslant 1 .
$$

Rearranging the items of $\rho$ into $v$ (see Appendix A) shows us that the matrix $\mathcal{L}$ is block diagonal. This is due to the fact that we have assumed the coefficients of $H$ and $T$ to be real numbers. This allows us to separate the system of differential equations into two subsystems that evolve independently, one involving the variables $\left\{\rho_{1,1}, \quad \rho_{2,2}, \quad \rho_{3,3}, \rho_{4,4}, a_{1,2}, a_{3,4}, \quad b_{1,3}, \quad b_{1,4}, b_{2,3}, \quad b_{2,4}\right\}$, and the other one involving the variables $\left\{a_{1,3}, a_{1,4}, \quad a_{2,3}, a_{2,4}, \quad b_{1,2}, b_{3,4}\right\}$ (see Appendix A for their definitions), which are the following:

$$
\begin{aligned}
\dot{\rho}_{1,1}= & -2 h_{1}(1-p) b_{1,3}-2 h_{2}(1-p) b_{1,4} \\
& +p\left(t_{1} \rho_{3,3}+t_{2} \rho_{4,4}\right)-p \rho_{1,1}, \\
\dot{\rho}_{2,2}= & -2 h_{3}(1-p) b_{2,3}-2 h_{4}(1-p) b_{2,4} \\
& +p\left[\left(1-t_{1}\right) \rho_{3,3}+\left(1-t_{2}\right) \rho_{4,4}\right]-p \rho_{2,2}, \\
\dot{\rho}_{3,3}= & 2 h_{1}(1-p) b_{1,3}+2 h_{3}(1-p) b_{2,3} \\
& +p\left(t_{3} \rho_{1,1}+t_{4} \rho_{2,2}\right)-(2+p) \rho_{3,3}, \\
\dot{\rho}_{4,4}= & 2 h_{2}(1-p) b_{1,4}+2 h_{4}(1-p) b_{2,4} \\
& +p\left[\left(1-t_{3}\right) \rho_{1,1}+\left(1-t_{4}\right) \rho_{2,2}\right]-(p+2) \rho_{4,4}, \\
\dot{a}_{1,2}= & -(1-p)\left(h_{3} b_{1,3}+h_{4} b_{1,4}+h_{1} b_{2,3}+h_{2} b_{2,4}\right) \\
& -p a_{1,2}, \\
\dot{b}_{1,3}= & (1-p)\left[h_{3} a_{1,2}-h_{2} a_{3,4}-h_{1}\left(\rho_{3,3}-\rho_{1,1}\right)\right] \\
& -(p+1) b_{1,3}, \\
\dot{b}_{1,4}= & (1-p)\left[h_{4} a_{1,2}-h_{1} a_{3,4}-h_{2}\left(\rho_{4,4}-\rho_{1,1}\right)\right] \\
& -(p+1) b_{1,4},
\end{aligned}
$$

$$
\begin{aligned}
\dot{b}_{2,3}= & (1-p)\left[h_{1} a_{1,2}-h_{4} a_{3,4}+h_{3}\left(\rho_{2,2}-\rho_{3,3}\right)\right] \\
& -(p+1) b_{2,3}, \\
\dot{b}_{2,4}= & (1-p)\left[h_{2} a_{1,2}-h_{3} a_{3,4}+h_{4}\left(\rho_{2,2}-\rho_{4,4}\right)\right] \\
& -(p+1) b_{2,4}, \\
\dot{a}_{3,4}= & (1-p)\left(h_{2} b_{1,3}+h_{1} b_{1,4}+h_{4} b_{2,3}+h_{3} b_{2,4}\right) \\
& -(p+2) a_{3,4},
\end{aligned}
$$

and

$$
\begin{aligned}
\dot{a}_{1,3}= & -(1-p)\left(h_{3} b_{1,2}+h_{2} b_{3,4}\right)-(p+1) a_{1,3}, \\
\dot{a}_{1,4}= & -(1-p)\left(h_{4} b_{1,2}-h_{1} b_{3,4}\right)-(p+1) a_{1,4}, \\
\dot{a}_{2,3}= & (1-p)\left(h_{1} b_{1,2}-h_{4} b_{3,4}\right)-(p+1) a_{2,3}, \\
\dot{a}_{2,4}= & (1-p)\left(h_{2} b_{1,2}+h_{3} b_{3,4}\right)-(p+1) a_{2,4}, \\
\dot{b}_{1,2}= & (1-p)\left(h_{3} a_{1,3}+h_{4} a_{1,4}-h_{1} a_{2,3}-h_{2} a_{2,4}\right) \\
& -p b_{1,2}, \\
\dot{b}_{3,4}= & (1-p)\left(h_{2} a_{1,3}-h_{1} a_{1,4}+h_{4} a_{2,3}-h_{3} a_{2,4}\right) \\
& -(p+2) b_{3,4} .
\end{aligned}
$$

In the first subsystem of differential equations the sinker nodes appear, but none of them are present in the second subsystem. This means that even if both subsystems may have a not-null initial value in the variables $\rho_{1,1}, \rho_{2,2}, a_{1,2}, b_{1,2}$, only the components $\rho_{1,1}, \rho_{2,2}, a_{1,2}$ may end up in the sink. The component $b_{1,2}$ of the initial state, which corresponds to the $r_{y}$ coordinates, will not contribute to the sink population, regardless of the entries in $H, T, p$. Potential differences in this component between the initial quantum states, which could help the discrimination, will not be visible at the sinks, effectively reducing the probability of correct decision. The value of $b_{1,2}$ of the initial quantum state is hence irrelevant to the discrimination performed on the sink nodes, and the problem is equivalent to discriminating the quantum states with this entry set to zero. In the literature, this phenomenon is explained in terms of invariant subspaces [24,25], that is, a subspace that prevents the dynamics to escape from the network. When this invariant subspace does not contain any sink, and it is initialized by the quantum states, it reduces the probability of correct detection since its time evolution is irrelevant for the discrimination.

Note that the presence of the invariant subspace is due to the topology of the $2 r-2 r-2$ model. The model $2-2-2$ instead shows a greater connectivity between the nodes and does not exhibit the separation of the ordinary differential equation system into two subsystems that generate the invariant subspace.

\section{APPENDIX C: SOLUTION OF THE MASTER EQUATION FOR $\boldsymbol{p}=\mathbf{0}$}

In this Appendix we solve the discrimination problem with $p=0$ in the case of equal probable pure states. Since $p=0$, there are no Lindblad operators in the master equation except the sink terms, i.e., vanishing terms (A4) and (A5), and the solution can be obtained by finding the optimal value of $h_{1}, h_{2}, h_{3}, h_{4}$. 
Since the term $b_{1,2}$ does not contribute to the discrimination, we will ignore the system of differential equations (B4) of the invariant subspace and focus on the other system of differential equations, (B3). We can then restrict our attention to the discrimination of pure states, such as

$$
\begin{aligned}
\left|\psi^{(1)}\right\rangle & =\left(\begin{array}{c}
\cos (\alpha) \\
\sin (\alpha)
\end{array}\right), \quad\left|\psi^{(2)}\right\rangle=\left(\begin{array}{c}
\cos (\beta) \\
\sin (\beta)
\end{array}\right), \\
\alpha & \neq \beta \in\left[0, \frac{\pi}{2}\right],
\end{aligned}
$$

which have no $r_{y}=\left\langle\psi^{(x)}\left|\sigma_{y}\right| \psi^{(x)}\right\rangle$ coordinates.

It is convenient to apply a rotation to the quantum states in order to highlight the symmetry of the problem. By applying the unitary

$$
U=\left(\begin{array}{cc}
\cos \left(\frac{\alpha+\beta}{2}\right) & \sin \left(\frac{\alpha+\beta}{2}\right) \\
-\sin \left(\frac{\alpha+\beta}{2}\right) & \cos \left(\frac{\alpha+\beta}{2}\right)
\end{array}\right)
$$

we obtain

$$
\begin{gathered}
\left|\phi^{(1)}\right\rangle=U\left|\psi^{(1)}\right\rangle=\left(\begin{array}{c}
\cos (\theta) \\
\sin (\theta)
\end{array}\right), \\
\left|\phi^{(2)}\right\rangle=U\left|\psi^{(2)}\right\rangle=\left(\begin{array}{c}
\cos (\theta) \\
-\sin (\theta)
\end{array}\right)
\end{gathered}
$$

with $\theta=(\alpha-\beta) / 2$.

We can then proceed to solve the discrimination problem on $\left|\phi^{(1)}\right\rangle,\left|\phi^{(2)}\right\rangle$. From its solution $H^{*}(\theta)$ we can recover the solution of the original problem as $H^{*}(\alpha, \beta)=U^{\dagger} H^{*}(\theta) U$.

We also assume that the optimal solution $H^{*}$ verifies $h_{1}=$ $h, h_{2}=h, h_{3}=h, h_{4}=-h$,

$$
H^{*(p=0)}=\left(\begin{array}{cccc}
0 & 0 & h & h \\
0 & 0 & h & -h \\
h & h & 0 & 0 \\
h & -h & 0 & 0
\end{array}\right)
$$

We will shortly see that under this ansatz we can optimize $h$ such that for $\tau$ going to infinity we reach the Helstrom bound.

Under these assumptions, the system of differential equation (B3) separates into two subsystems with disjoint variables. For instance, $\rho_{3,3}, s=b_{1,3}+b_{2,3}, \quad p=\rho_{1,1}+\rho_{2,2}+$ $2 a_{1,2}$ form the following system of differential equations:

$$
\begin{aligned}
\dot{\rho}_{3,3} & =-2 \rho_{3,3}+2 h s, \\
\dot{s} & =-2 h \rho_{3,3}-s+h p, \\
\dot{p} & =-4 h s .
\end{aligned}
$$

The same system holds for the variables $\rho_{4,4}, d=b_{1,4}-$ $b_{2,4}, \quad m=\rho_{1,1}+\rho_{2,2}-2 a_{1,2}$ in place of $\rho_{3,3}, s, \quad p$ respectively. In matrix form,

$$
\dot{w}=\left[\begin{array}{ccc}
-2 & 2 h & 0 \\
-2 h & -1 & h \\
0 & -4 h & 0
\end{array}\right] w
$$

with $w=\left[\rho_{3,3} s p\right]^{T}$ or $w=\left[\rho_{4,4} d m\right]^{T}$.
A fundamental set of solutions for the system (C7) can be arranged in a matrix as

$$
W(t)=e^{-t}\left(\begin{array}{ccc}
2 h & \left(1-4 h^{2}+z\right) e^{-z t} & \left(1-4 h^{2}-z\right) e^{z t} \\
1 & 2 h(1+z) e^{-z t} & 2 h(1-z) e^{z t} \\
4 h & 8 h^{2} e^{-z t} & 8 h^{2} e^{z t}
\end{array}\right)
$$

with $z=\sqrt{1-8 h^{2}}$. The Wronskian reads $\operatorname{Det}[W(t)]=$ $-16 h^{2}\left(1-8 h^{2}\right)^{3 / 2} e^{-3 t}$.

The initial conditions for $(\mathrm{C} 7)$ are

$$
\begin{aligned}
\rho_{3,3}(0) & =0, \\
s(0) & =b_{1,3}(0)+b_{2,3}(0)=0, \\
p(0) & =\rho_{1,1}(0)+\rho_{2,2}(0)+2 a_{1,2}(0) \\
& =1+(-1)^{x} \sin (2 \theta),
\end{aligned}
$$

with $x=0,1$ denoting the initial quantum state. Similarly, we have

$$
\begin{aligned}
\rho_{4,4}(0) & =0, \\
d(0) & =b_{1,4}(0)-b_{2,4}(0)=0, \\
m(0) & =\rho_{1,1}(0)+\rho_{2,2}(0)-2 a_{1,2}(0) \\
& =1-(-1)^{x} \sin (2 \theta) .
\end{aligned}
$$

In particular, defining $\rho_{n, n}^{(x)}(t)=\operatorname{Tr}\left[\Pi_{n} \rho^{(x)}(t)\right], \quad \rho^{(x)}(0)=$ $\left|\phi^{(x)}\right\rangle\left\langle\phi^{(x)}\right|, x=1,2$, we obtain

$$
\begin{aligned}
& \rho_{3,3}^{(x)}(t)=\frac{4 h^{2} e^{-t} \sinh ^{2}\left(\frac{1}{2} z t\right)}{z^{2}}\left[1+(-1)^{x} \sin (2 \theta)\right], \\
& \rho_{4,4}^{(x)}(t)=\frac{4 h^{2} e^{-t} \sinh ^{2}\left(\frac{1}{2} z t\right)}{z^{2}}\left[1-(-1)^{x} \sin (2 \theta)\right] .
\end{aligned}
$$

To solve the discrimination problem, we need to maximize the probability of correct decision. This can be written as

$$
\begin{aligned}
P_{c}^{(p=0)}(\tau)= & \int_{0}^{\tau} \rho_{3,3}^{(1)}(t)+\rho_{4,4}^{(2)}(t) d t \\
= & \frac{1+\sin (2 \theta)}{2} \\
& \times\left\{1-e^{-\tau}\left[\frac{z \sinh (z \tau)+\cosh (z \tau)-1}{z^{2}}+1\right]\right\} .
\end{aligned}
$$

It is clear that in order to maximize $P_{c}^{(p=0)}(\tau)$ we need to minimize the term $f(z)=[z \sinh (z \tau)+\cosh (z \tau)-1] / z^{2}, z=$ $\sqrt{1-8 h^{2}}$. Unfortunately, this transcendental real function in the complex variable $z$ cannot be minimized analytically, and we need to resort to numerical methods. Graphically, we can see that the global minimum is located in the region where $1-8 h^{2}<0$, meaning that $z$ is a pure imaginary number. Writing $z=i \xi / \tau$, the function to minimize becomes $f(\xi)=$ $\tau\left[\frac{\sin \xi}{\xi}+\tau \frac{1-\cos \xi}{\xi^{2}}\right]$. Both $f_{1}(\xi)=\sin (\xi) / \xi$ and $f_{2}(\xi)=[1-$ $\cos (\xi)] / \xi^{2}$ are oscillating functions with the global point of minimum corresponding to the first local minimum, and the same holds for $f(\xi)$, with the point of minimum located close to those of $f_{1}(\xi)$ or $f_{2}(\xi)$ depending on $\tau$ (see Fig. 10).

The asymptotic probability of correct decision is obtained from (C13) for $\tau \rightarrow \infty$. In this case the term in brackets vanished, and we obtain the Helstrom bound (11) independently 


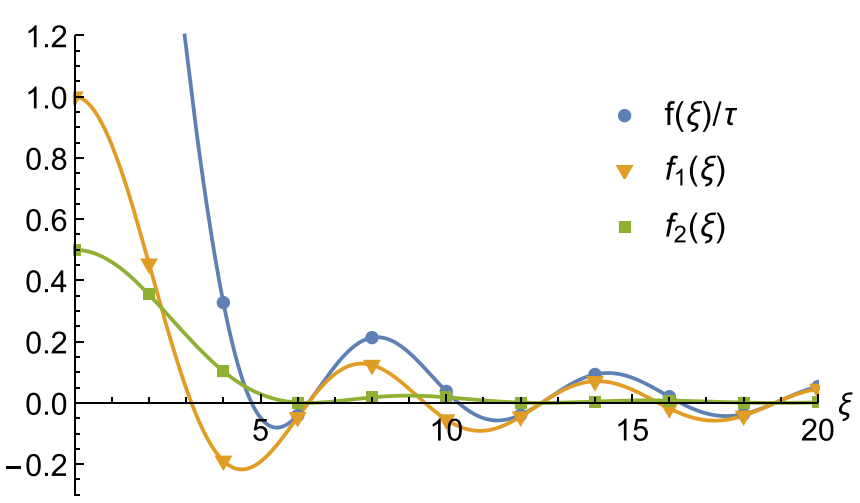

FIG. 10. Plot of the term $f(\xi) / \tau$ to minimize (blue line, circle markers), along with the terms $f_{1}(\xi)$ (orange line, triangular markers) and $f_{2}(\xi)$ (green line, square markers). Both $f_{1}(\xi)$ and $f_{2}(\xi)$ are oscillating functions with the point of minimum corresponding to the first local minimum. The global minimum of $f(\xi)$ is between these two minimum, close to the minimum of $f_{1}(\xi)$ for $\tau \approx 0$, or the minimum of $f_{2}(\xi)$ for $\tau \gg 1$. The total evolution time is $\tau=5$.

of the value $h$, as long as the optimal Hamiltonian operator verifies (C5).

\section{APPENDIX D: SOLUTION OF THE MASTER EQUATION FOR $p=1$}

In this Appendix we solve the discrimination problem with $p=1$. In this case, the Hamiltonian operator no longer contributes to the evolution of the quantum system since the term (A3) vanishes. We obtain hence a classical random walk, which can be optimized for the entries of $T$,

$$
T=\left(\begin{array}{cccc}
0 & 0 & \frac{1}{2}+d_{1} & \frac{1}{2}-d_{2} \\
0 & 0 & \frac{1}{2}-d_{1} & \frac{1}{2}+d_{2} \\
\frac{1}{2}+d_{3} & \frac{1}{2}-d_{4} & 0 & 0 \\
\frac{1}{2}-d_{3} & \frac{1}{2}+d_{4} & 0 & 0
\end{array}\right) .
$$

On the variables $d_{k}$ it holds the constraints

$$
-1 / 2 \leqslant d_{k} \leqslant 1 / 2, \quad k=1, \ldots, 4 .
$$

With $p=1$, not only does the system of differential equations separate into the subsystems (B3) and (B4), but the coherence components $a_{i, j}, b_{i, j}$ also evolve independently while the diagonal terms form the coupled system

$$
\dot{v}=\left[\begin{array}{cccc}
-1 & 0 & \frac{1}{2}+d_{1} & \frac{1}{2}-d_{2} \\
0 & -1 & \frac{1}{2}-d_{1} & \frac{1}{2}+d_{2} \\
\frac{1}{2}+d_{3} & \frac{1}{2}-d_{4} & -3 & 0 \\
\frac{1}{2}-d_{3} & \frac{1}{2}+d_{4} & 0 & -3
\end{array}\right] v
$$

with $v=\left[\begin{array}{llll}\rho_{1,1}, & \rho_{2,2}, & \rho_{3,3}, & \rho_{4,4}\end{array}\right]^{T}$. As in the case $p=0$, we can evaluate the fundamental set of solutions and the Wronskian of the system. The initial conditions for the system (D3)

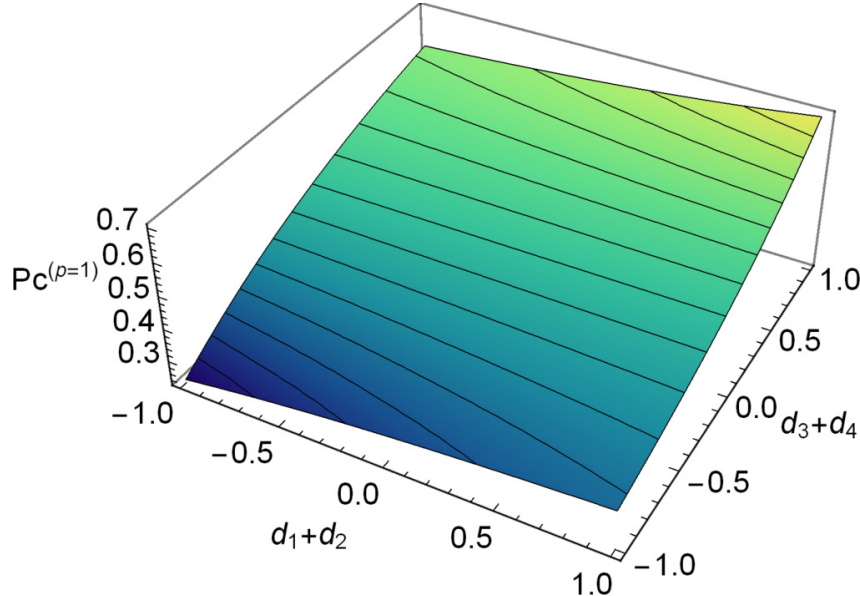

FIG. 11. Probability $P_{c}^{(p=1)}(\tau)$ as a function of $d_{1}+d_{2}$ and $d_{3}+$ $d_{4}$ in the case of $\Delta \rho_{2}-\Delta \rho_{1}=0.5, \tau=5$. The black lines are contour lines, and the maximum is obtained for $d_{1}+d_{2}=d_{3}+d_{4}=$ $\operatorname{sgn}\left(\Delta \rho_{2}-\Delta \rho_{1}\right)$. The same behavior is exhibited for all $\tau$, with the surface being flatter for $\tau \approx 0$, while for $\tau \rightarrow \infty$ the surface is practically indistinguishable form the plotted one.

are defined from the diagonal entries of the initial states $\rho_{1}$ and $\rho_{2}$. We then obtain the solutions $\rho_{3,3}^{(1)}(t)$ and $\rho_{4,4}^{(2)}(t)$ reported in Eqs. (D6) and (D7), with the corresponding probability of correct decision evaluated in Eq. (D8). As we can see, this probability depends on $\Delta \rho_{2}-\Delta \rho_{1}, \Delta \rho_{x}=\frac{\rho_{1,1}^{(1)}(0)-\rho_{2,2}^{(2)}(0)}{2}$, as well as $s_{12}=d_{1}+d_{2}$ and $s_{34}=d_{3}+d_{4}$. Notice the symmetry in $d_{1}, d_{2}\left[d_{3}, d_{4}\right]$. We can maximize $P_{c}^{(p=1)}(\tau)$ with respect to $d_{1}+d_{2}$ and $d_{3}+d_{4}$ (see Fig. 11), and the optimal solution is $d_{1}+d_{2}=d_{3}+d_{4}=\operatorname{sgn}\left(\Delta \rho_{2}-\Delta \rho_{1}\right)$ obtained for $d_{1}=$ $d_{2}=d_{3}=d_{4}=\frac{1}{2} \operatorname{sgn}\left(\Delta \rho_{2}-\Delta \rho_{1}\right)$. For instance, in the case of $\Delta \rho_{2}>\Delta \rho_{1}$, the resulting optimal matrix $T^{*}$ reads

$$
T^{*}=\left(\begin{array}{llll}
0 & 0 & 1 & 0 \\
0 & 0 & 0 & 1 \\
1 & 0 & 0 & 0 \\
0 & 1 & 0 & 0
\end{array}\right)
$$

Asymptotically, for $\tau \rightarrow \infty$ the optimal probability of correct decision becomes

$$
P_{c}^{*(p=1)}(\infty)=\frac{1+\left|\Delta \rho_{2}-\Delta \rho_{1}\right|}{2},
$$

which coincides with the Helstrom bound for the discrimination of the quantum states $\rho^{(1, \text { class. })}$ and $\rho^{(2, \text { class. })}$, obtained from $\rho^{(1)}, \rho^{(2)}$ by removing the coherences, effectively turning a quantum state into a classical one:

$$
\begin{aligned}
\rho_{3,3}^{(x)}(t)= & e^{-2 t}\left\{\frac{\left(d_{3}+d_{4}\right)\left[d_{1}-d_{2}+\left(d_{1}+d_{2}\right)\left(d_{3}-d_{4}\right)\right] \sinh \left[\sqrt{1+\left(d_{1}+d_{2}\right)\left(d_{3}+d_{4}\right)} t\right]}{2\left[1-\left(d_{1}+d_{2}\right)\left(d_{3}+d_{4}\right)\right] \sqrt{1+\left(d_{1}+d_{2}\right)\left(d_{3}+d_{4}\right)}}\right. \\
& \left.+\frac{\left[1+\left(1-2 d_{2}\right) d_{3}-\left(1+2 d_{2}\right) d_{4}\right] \sinh (\sqrt{2} t)}{2 \sqrt{2}\left[1-\left(d_{1}+d_{2}\right)\left(d_{3}+d_{4}\right)\right]}+\frac{\left(d_{3}+d_{4}\right) \sinh \left[\sqrt{1+\left(d_{1}+d_{2}\right)\left(d_{3}+d_{4}\right)} t\right]}{\sqrt{1+\left(d_{1}+d_{2}\right)\left(d_{3}+d_{4}\right)}} \Delta \rho_{x}\right\},
\end{aligned}
$$




$$
\begin{aligned}
\rho_{4,4}^{(x)}(t)= & e^{-2 t}\left\{-\frac{\left(d_{3}+d_{4}\right)\left[d_{1}-d_{2}+\left(d_{1}+d_{2}\right)\left(d_{3}-d_{4}\right)\right] \sinh \left[\sqrt{1+\left(d_{1}+d_{2}\right)\left(d_{3}+d_{4}\right)} t\right]}{2\left(1-\left(d_{1}+d_{2}\right)\left(d_{3}+d_{4}\right)\right) \sqrt{1+\left(d_{1}+d_{2}\right)\left(d_{3}+d_{4}\right)}}\right. \\
+ & \left.\frac{\left[1-\left(1+2 d_{1}\right) d_{3}+\left(1-2 d_{1}\right) d_{4}\right] \sinh (\sqrt{2} t)}{2 \sqrt{2}\left[1-\left(d_{1}+d_{2}\right)\left(d_{3}+d_{4}\right)\right]}+\frac{\left(d_{3}+d_{4}\right) \sinh \left[\sqrt{1+\left(d_{1}+d_{2}\right)\left(d_{3}+d_{4}\right)} t\right]}{\sqrt{1+\left(d_{1}+d_{2}\right)\left(d_{3}+d_{4}\right)}} \Delta \rho_{x}\right\}, \\
P_{c}^{(p=1)}(\tau)= & \frac{1}{2}+\frac{(\sqrt{2}-1) e^{-(2+\sqrt{2}) \tau}-(\sqrt{2}+1) e^{-(2-\sqrt{2}) \tau}}{4}+\frac{\left(d_{3}+d_{4}\right)\left(\Delta \rho_{2}-\Delta \rho_{1}\right)}{3-\left(d_{1}+d_{2}\right)\left(d_{3}+d_{4}\right)} \\
& +\frac{\left(d_{3}+d_{4}\right)\left(\Delta \rho_{2}-\Delta \rho_{1}\right)}{\sqrt{1+\left(d_{1}+d_{2}\right)\left(d_{3}+d_{4}\right)}}\left\{\frac{e^{-\left[2+\sqrt{1+\left(d_{1}+d_{2}\right)\left(d_{3}+d_{4}\right)} \tau\right.}}{\sqrt{1+\left(d_{1}+d_{2}\right)\left(d_{3}+d_{4}\right)}+2}+\frac{e^{-\left[2-\sqrt{1+\left(d_{1}+d_{2}\right)\left(d_{3}+d_{4}\right)}\right] \tau}}{\sqrt{1+\left(d_{1}+d_{2}\right)\left(d_{3}+d_{4}\right)}-2}\right\} .
\end{aligned}
$$

[1] J. D. Whitfield, C. A. Rodríguez-Rosario, and A. AspuruGuzik, Quantum stochastic walks: A generalization of classical random walks and quantum walks, Phys. Rev. A 81, 022323 (2010).

[2] Y. Aharonov, L. Davidovich, and N. Zagury, Quantum random walks, Phys. Rev. A 48, 1687 (1993).

[3] J. Kempe, Quantum random walks: An introductory overview, Contemp. Phys. 44, 307 (2003).

[4] V. Kendon, Decoherence in quantum walks-A review, Math. Struct. Comput. Sci. 17, 1169 (2007).

[5] S. E. Venegas-Andraca, Quantum walks: A comprehensive review, Quant. Info. Proc. 11, 1015 (2012).

[6] D. Reitzner, D. Nagaj, and V. Buzek, Quantum walks, Acta Phys. Slovaca 61, 603 (2011).

[7] A. M. Childs, Universal Computation by Quantum Walk, Phys. Rev. Lett. 102, 180501 (2009).

[8] A. M. Childs, D. Gosset, and Z. Webb, Universal computation by multiparticle quantum walk, Science 339, 791 (2013).

[9] N. B. Lovett, S. Cooper, M. Everitt, M. Trevers, and V. Kendon, Universal quantum computation using the discrete-time quantum walk, Phys. Rev. A 81, 042330 (2010).

[10] A. Amainis, Quantum walks and their algorithmic applications, Int. J. Quantum. Inform. 01, 507 (2003).

[11] A. Ambainis, Quantum random walks-New method for designing quantum algorithms, in SOFSEM 2008: Theory and Practice of Computer Science, edited by V. Geffert, J. Karhumäki, A. Bertoni, B. Preneel, P. Návrat, and M. Bieliková (Springer, Berlin, 2008), pp. 1-4.

[12] A. Ambainis, New developments in quantum algorithms, in Mathematical Foundations of Computer Science 2010, edited by P. Hliněný and A. Kučera (Springer, Berlin, 2010), pp. 1-11.

[13] P. R. Giri and V. E. Korepin, A review on quantum search algorithms, Quant. Info. Proc. 16, 315 (2017).

[14] M. Santha, Quantum walk based search algorithms, in Theory and Applications of Models of Computation, edited by M. Agrawal, D. Du, Z. Duan, and A. Li (Springer, Berlin, 2008), pp. 31-46.

[15] E. Farhi and S. Gutmann, Quantum computation and decision trees, Phys. Rev. A 58, 915 (1998).

[16] A. M. Childs, R. Cleve, E. Deotto, E. Farhi, S. Gutmann, and D. A. Spielman, Exponential algorithmic speedup by a quantum walk, in Proceedings of the Thirty-fifth Annual ACM Symposium on Theory of Computing, STOC '03 (ACM, New York, 2003), pp. 59-68.
[17] N. Shenvi, J. Kempe, and K. Birgitta Whaley, Quantum random-walk search algorithm, Phys. Rev. A 67, 052307 (2003).

[18] A. M. Childs and J. Goldstone, Spatial search by quantum walk, Phys. Rev. A 70, 022314 (2004).

[19] A. Ambainis, J. Kempe, and A. Rivosh, Coins make quantum walks faster, in Proceedings of the Sixteenth Annual ACM-SIAM Symposium on Discrete Algorithms, SODA '05 (Society for Industrial and Applied Mathematics, Philadelphia, 2005), pp. 1099-1108.

[20] M. B. Plenio and S. F. Huelga, Dephasing-assisted transport: Quantum networks and biomolecules, New J. Phys. 10, 113019 (2008).

[21] J. J. Mendoza-Arenas, T. Grujic, D. Jaksch, and S. R. Clark, Dephasing enhanced transport in nonequilibrium strongly correlated quantum systems, Phys. Rev. B 87, 235130 (2013).

[22] L. D. Contreras-Pulido, M. Bruderer, S. F. Huelga, and M. B. Plenio, Dephasing-assisted transport in linear triple quantum dots, New J. Phys. 16, 113061 (2014).

[23] A. Olaya-Castro, C. F. Lee, F. F. Olsen, and N. F. Johnson, Efficiency of energy transfer in a light-harvesting system under quantum coherence, Phys. Rev. B 78, 085115 (2008).

[24] F. Caruso, A. W. Chin, A. Datta, S. F. Huelga, and M. B. Plenio, Highly efficient energy excitation transfer in light-harvesting complexes: The fundamental role of noise-assisted transport, J. Chem. Phys. 131, 105106 (2009).

[25] A. W. Chin, A. Datta, F. Caruso, S. F. Huelga, and M. B. Plenio, Noise-assisted energy transfer in quantum networks and lightharvesting complexes, New J. Phys. 12, 065002 (2010).

[26] F. Caruso, A. W. Chin, A. Datta, S. F. Huelga, and M. B. Plenio, Entanglement and entangling power of the dynamics in light-harvesting complexes, Phys. Rev. A 81, 062346 (2010).

[27] E. Sánchez-Burillo, J. Duch, J. Gómez-Gardeñes, and D. Zueco, Quantum navigation and ranking in complex networks, Sci. Rep. 2, 605 EP (2012).

[28] C. Liu and R. Balu, Steady states of continuous-time open quantum walks, Quant. Info. Proc. 16, 173 (2017).

[29] A. Glos, J. A. Miszczak, and M. Ostaszewski, Limiting properties of stochastic quantum walks on directed graphs, J. Phys. A: Math. Theor. 51, 035304 (2017).

[30] K. Domino, A. Glos, and M. Ostaszewski, Superdiffusive quantum stochastic walk definable on arbitrary directed graph, Quant. Info. Comput. 17, 973 (2017). 
[31] K. Domino, A. Glos, M. Ostaszewski, Ł. Pawela, and P. Sadowski, Properties of quantum stochastic walks from the asymptotic scaling exponent, Quant. Info. Comput. 18, 181 (2018).

[32] M. Schuld, I. Sinayskiy, and F. Petruccione, Quantum walks on graphs representing the firing patterns of a quantum neural network, Phys. Rev. A 89, 032333 (2014).

[33] M. Mohseni, P. Rebentrost, S. Lloyd, and A. Aspuru-Guzik, Environment-assisted quantum walks in photosynthetic energy transfer, J. Chem. Phys. 129, 174106 (2008).

[34] F. Caruso, Universally optimal noisy quantum walks on complex networks, New J. Phys. 16, 055015 (2014).

[35] S. Viciani, M. Lima, M. Bellini, and F. Caruso, Observation of Noise-Assisted Transport in an All-Optical Cavity-Based Network, Phys. Rev. Lett. 115, 083601 (2015).

[36] F. Caruso, A. Crespi, A. G. Ciriolo, F. Sciarrino, and R. Osellame, Fast escape of a quantum walker from an integrated photonic maze, Nat. Commun. 7, 11682 (2016).

[37] H. Park, N. Heldman, P. Rebentrost, L. Abbondanza, A. Iagatti, A. Alessi, B. Patrizi, M. Salvalaggio, L. Bussotti, M. Mohseni et al., Enhanced energy transport in genetically engineered excitonic networks, Nat. Mater. 15, 211 EP (2015).

[38] A. Kossakowski, On quantum statistical mechanics of nonHamiltonian systems, Rep. Math. Phys. 3, 247 (1972).

[39] G. Lindblad, On the generators of quantum dynamical semigroups, Commun. Math. Phys. 48, 119 (1976).

[40] V. Gorini, A. Kossakowski, and E. C. G. Sudarshan, Completely positive dynamical semigroups of $N$-level systems, J. Math. Phys. 17, 821 (1976).

[41] C. Helstrom, Quantum Detection and Estimation Theory, Mathematics in Science and Engineering, Vol. 123 (Academic Press, New York, 1976).

[42] A. S. Holevo, Statistical problems in quantum physics, in Proceedings of the Second Japan-USSR Symposium on Probability Theory, edited by G. Maruyama and Y. V. Prokhorov (Springer, Berlin, 1973), pp. 104-119.

[43] H. Yuen, R. Kennedy, and M. Lax, Optimum testing of multiple hypotheses in quantum detection theory, IEEE Trans. Inf. Theory 21, 125 (1975).

[44] A. S. Kholevo, On asymptotically optimal hypothesis testing in quantum statistics, Theory Probab. Appl. 23, 411 (1979).

[45] A. Chefles, Quantum state discrimination, Contemp. Phys. 41, 401 (2000).

[46] J. A. Bergou, Quantum state discrimination and selected applications, J. Phys.: Conf. Ser. 84, 012001 (2007).

[47] J. A. Bergou, Discrimination of quantum states, J. Mod. Opt. 57, 160 (2010).

[48] S. M. Barnett and S. Croke, Quantum state discrimination, Adv. Opt. Photon. 1, 238 (2009).

[49] D. Sych and G. Leuchs, Practical Receiver for Optimal Discrimination of Binary Coherent Signals, Phys. Rev. Lett. 117, 200501 (2016).

[50] S. Croke, S. M. Barnett, and G. Weir, Optimal sequential measurements for bipartite state discrimination, Phys. Rev. A 95, 052308 (2017).

[51] M. Rosati, G. De Palma, A. Mari, and V. Giovannetti, Optimal quantum state discrimination via nested binary measurements, Phys. Rev. A 95, 042307 (2017).

[52] G. Weir, S. M. Barnett, and S. Croke, Optimal discrimination of single-qubit mixed states, Phys. Rev. A 96, 022312 (2017).
[53] M. Namkung and Y. Kwon, Sequential state discrimination of coherent states, Sci. Rep. 8, 16915 (2018).

[54] K. Flatt, S. M. Barnett, and S. Croke, Multiple-copy state discrimination of noisy qubits, Phys. Rev. A 100, 032122 (2019).

[55] C. R. Müller, M. A. Usuga, C. Wittmann, M. Takeoka, C. Marquardt, U. L. Andersen, and G. Leuchs, Quadrature phase shift keying coherent state discrimination via a hybrid receiver, New J. Phys. 14, 083009 (2012).

[56] M. A. Solís-Prosser, M. F. Fernandes, O. Jiménez, A. Delgado, and L. Neves, Experimental Minimum-Error Quantum-State Discrimination in High Dimensions, Phys. Rev. Lett. 118, 100501 (2017).

[57] M. T. DiMario and F. E. Becerra, Robust Measurement for the Discrimination of Binary Coherent States, Phys. Rev. Lett. 121, 023603 (2018).

[58] S. Izumi, J. S. Neergaard-Nielsen, and U. L. Andersen, Tomography of a displacement photon counter for discrimination of single-rail optical qubits, J. Phys. B: At. Mol. Opt. Phys. 51, 085502 (2018).

[59] R. Han, J. A. Bergou, and G. Leuchs, Near optimal discrimination of binary coherent signals via atom-light interaction, New J. Phys. 20, 043005 (2018).

[60] K. Nakahira, K. Kato, and T. S. Usuda, Optimal discrimination of optical coherent states cannot always be realized by interfering with coherent light, photon counting, and feedback, Phys. Rev. A 97, 022320 (2018).

[61] M. Fanizza, A. Mari, and V. Giovannetti, Optimal universal learning machines for quantum state discrimination, IEEE Trans. Inf. Theory 65, 5931 (2019).

[62] P. Kurzyński and A. Wójcik, Quantum Walk as a Generalized Measuring Device, Phys. Rev. Lett. 110, 200404 (2013).

[63] Z. Li, H. Zhang, and H. Zhu, Implementation of generalized measurements on a qudit via quantum walks, Phys. Rev. A 99, 062342 (2019).

[64] C. M. Bishop, Pattern Recognition and Machine Learning, Information Science and Statistics (Springer-Verlag, Berlin, 2006).

[65] I. Goodfellow, Y. Bengio, and A. Courville, Deep Learning (MIT Press, Cambridge, MA, 2016) http: //www.deeplearningbook.org

[66] T. Hastie, R. Tibshirani, and J. Friedman, The Elements of Statistical Learning, Springer Series in Statistics (Springer, New York, 2001)

[67] Y. C. Eldar, A. Megretski, and G. C. Verghese, Designing optimal quantum detectors via semidefinite programming, IEEE Trans. Inf. Theory 49, 1007 (2003).

[68] Y. C. Eldar, A. Megretski, and G. C. Verghese, Optimal detection of symmetric mixed quantum states, IEEE Trans. Inf. Theory 50, 1198 (2004).

[69] K. Nakahira and T. S. Usuda, Quantum measurement for a group-covariant state set, Phys. Rev. A 87, 012308 (2013).

[70] N. Dalla Pozza and G. Pierobon, Optimality of square-root measurements in quantum state discrimination, Phys. Rev. A 91, 042334 (2015).

[71] S. Boyd and L. Vandenberghe, Convex Optimization (Cambridge University Press, New York, 2004).

[72] R. Byrd, M. Hribar, and J. Nocedal, An interior point algorithm for large-scale nonlinear programming, SIAM J. Optim. 9, 877 (1999). 
[73] R. H. Byrd, J. C. Gilbert, and J. Nocedal, A trust region method based on interior point techniques for nonlinear programming, Math. Program. 89, 149 (2000).

[74] R. Waltz, J. Morales, J. Nocedal, and D. Orban, An interior algorithm for nonlinear optimization that combines line search and trust region steps, Math. Program. 107, 391 (2006).

[75] N. Dalla Pozza and M. G. A. Paris, Naimark extension for the single-photon canonical phase measurement, Phys. Rev. A 100, 032126 (2019).
[76] M. A. Neumark, On spectral functions of a symmetric operator, Izv. Akad. Nauk SSSR, Ser. Mat. 7, 285 (1943).

[77] N. Dalla Pozza and M. G. A. Paris, An effective iterative method to build the Naimark extension of rank- $n$ POVMs, Int. J. Quantum. Inform. 15, 1750029 (2017).

[78] J. R. Magnus and H. Neudecker, Matrix Differential Calculus with Applications in Statistics and Econometrics, 2nd ed. (John Wiley, New York, 1999). 